\title{
Subdivisions of Toric Complexes
}

\author{
MORTEN BRUN \\ brun@mathematik.uni-osnabrueck.de \\ FB Mathematik/Informatik, Universität Osnabrück, 49069 Osnabrück, Germany \\ TIM RÖMER \\ troemer@mathematik.uni-osnabrueck.de \\ FB Mathematik/Informatik, Universität Osnabrück, 49069 Osnabrück, Germany
}

Received March 8, 2004; Revised August 3, 2004; Accepted September 9, 2004

\begin{abstract}
We introduce toric complexes as polyhedral complexes consisting of rational cones together with a set of integral generators for each cone, and we define their associated face rings. Abstract simplicial complexes and rational fans can be considered as toric complexes, and the face ring for toric complexes extends Stanley and Reisner's face ring for abstract simplicial complexes [20] and Stanley's face ring for rational fans [21]. Given a toric complex with defining ideal $I$ for the face ring we give a geometrical interpretation of the initial ideals of $I$ with respect to weight orders in terms of subdivisions of the toric complex generalizing a theorem of Sturmfels in [23]. We apply our results to study edgewise subdivisions of abstract simplicial complexes.
\end{abstract}

Keywords: initial ideal, toric ideal, polyhedral complex, regular subdivision, edgewise subdivision, face ring

\section{Introduction}

The aim of this paper is to define and study toric complexes. An embedded toric complex is a rational fan together with a distinguished set of generators, consisting of lattice points, for each cone. Since an abstract simplicial complex with $d$ vertices corresponds to a simplicial fan whose rays are spanned by the elements of the standard basis for $\mathbb{R}^{d}$, an abstract simplicial complex can be considered as an example of a toric complex. Generalizing the Stanley-Reisner ideal of a simplicial complex and the toric ideal of a configuration of lattice points, we establish a connection between binomial ideals in a polynomial ring and toric complexes. Our main result shows that a regular subdivision of a toric complex corresponds to the radical of an initial ideal of the associated binomial ideal. As an application of this correspondence we consider edgewise subdivisions of simplicial complexes and we describe the associated deformations of Veronese subrings of Stanley-Reisner rings.

Let us go more into detail. $K$ denotes a noetherian commutative ring. A finite subset $F$ of $\mathbb{Z}^{d}$ determines a (non-normal) affine toric variety $X_{F}=\operatorname{Spec}\left(K\left[M_{F}\right]\right)$. Here $M_{F}$ is the submonoid of $\mathbb{Z}^{d}$ generated by $F$ and $K\left[M_{F}\right]$ is the subring of $K\left[\mathbb{Z}^{d}\right]=$ $K\left[t_{1}, \ldots, t_{d}, t_{1}^{-1}, \ldots, t_{d}^{-1}\right]$ generated by monomials of the form $t^{a}=\prod_{i=1}^{d} t_{i}^{a_{i}}$ for $a=$ $\left(a_{1}, \ldots, a_{d}\right) \in F$. In the classical situation, where $M_{F}$ is the set of lattice points in the set cone $(F) \subseteq \mathbb{R}^{d}$ of positive real linear combinations of elements of $F$, the variety $X_{F}$ is a normal affine toric variety. A regular subdivision of cone $(F)$ supported on $F$, called projective subdivision in [15, p. 111], is a rational fan of the form $\Sigma=\{\operatorname{cone}(G): G \in \Pi\}$ for a set $\Pi$ of subsets of $F$ with cone $(F)=\cup_{G \in \Pi} \operatorname{cone}(G)$ satisfying that there exist linear 
forms $\alpha_{G}: \mathbb{R}^{d} \rightarrow \mathbb{R}$ such that the restrictions $\alpha_{G}: \operatorname{cone}(G) \rightarrow \mathbb{R}$ assemble to a continuous convex function $f: \operatorname{cone}(F) \rightarrow \mathbb{R}$. As explained for instance in [15, pp. 28-30] such regular subdivisions correspond to coherent sheaves on $X_{F}$, and there exists a regular subdivision of cone $(F)$ such that the fan $\Sigma$ defines a resolution of singularities $X(\Sigma) \rightarrow X_{F}$.

Our main result in Section 5 generalizes the correspondence established by Sturmfels in [22] and [23] between regular subdivisions of cone $(F)$ and initial ideals of the kernel $\mathcal{I}_{F}$ of the homomorphism from the polynomial ring $K[F]=K\left[x_{a}: a \in F\right]$ to $K\left[\mathbb{Z}^{d}\right]$ taking $x_{a}$ to $t^{a}$.

More precisely, a function $\omega: F \rightarrow \mathbb{R}$ defines a weight order on $K[F]$ with $\omega\left(x^{u}\right)=$ $\sum_{a \in F} u(a) \omega(a)$ for a monomial $x^{u}=\prod_{a \in F} x_{a}^{u(a)}$. The initial ideal $\operatorname{in}_{\omega}\left(\mathcal{I}_{F}\right)$ of $\mathcal{I}_{F}$ with respect to $\omega$ is the ideal generated by the initial polynomials $\operatorname{in}_{\omega}(f)=\sum_{\omega(u)=\omega(f)} f_{u} x^{u}$ where $f=\sum_{u} f_{u} x^{u}$ is non-zero in $K[F]$ and $\omega(f)=\max \left\{\omega(u) \in \mathbb{R}: f_{u} \neq 0\right\}$.

The function $\omega$ also defines a collection $\Pi_{\text {sd }_{\omega} T(F)}$ of subsets of $F$. Given $G \subseteq F$ let $C$ denote the minimal face of cone $(F)$ containing $G$ and let $G^{\prime}=F \cap C$. The subset $G$ of $F$ is in $\Pi_{\mathrm{sd}_{\omega} T(F)}$ if and only if there exists a linear form $\alpha_{G}$ on $\mathbb{R}^{d}$ with $\alpha_{G}(a) \leq \omega(a)$ for $a \in G^{\prime}$ such that equality holds precisely if $a \in G$. This collection $\Pi=\Pi_{\mathrm{sd}_{\omega} T(F)}$ of subsets of $\mathbb{Z}^{d}$ is an embedded toric complex $\operatorname{sd}_{\omega} T(F)$, that is, it satisfies firstly that for $H \subseteq G$ with $G \in \Pi$ we have that $H$ is in $\Pi$ if and only if cone $(H)$ is a face of cone $(G)$ with cone $(H) \cap G=H$ and secondly that if $G$ and $H$ are in $\Pi$, then their intersection is an element of $\Pi$. The fan $\Sigma=\{\operatorname{cone}(G): G \in \Pi\}$ is a regular partial subdivision of cone $(F)$ in the sense that the restrictions $\alpha_{G}$ : cone $(G) \rightarrow \mathbb{R}$ assemble to a continuous function $f: \cup_{G \in \Pi} \operatorname{cone}(G) \rightarrow \mathbb{R}$ whose restriction to any face of cone $(F)$ contained in $\cup_{G \in \Pi} \operatorname{cone}(G)$ is convex.

We carry the above idea one step further. Given an embedded toric complex $T$ consisting of the collection $\Pi_{T}$ of subsets of $\mathbb{Z}^{d}$ and a function of the form $\omega: \operatorname{Gen}(T)=\cup_{G \in \Pi_{T}} G \rightarrow$ $\mathbb{R}$, we have the embedded toric complex $\operatorname{sd}_{\omega} T(F)$ for every $F \in \Pi_{T}$. The embedded toric complex $\operatorname{sd}_{\omega} T$ is defined to consist of the collection $\Pi_{\operatorname{sd}_{\omega} T}=\bigcup_{F \in \Pi_{T}} \Pi_{\mathrm{sd}_{\omega} T(F)}$. This is the regular partial subdivision of $T$ induced by $\omega$.

In analogy with the Stanley-Reisner ideal of an abstract simplicial complex (see [20] for details) there is a square-free monomial ideal $J_{T}$ in $K[\operatorname{Gen}(T)]$ such that a monomial $x^{u}=\prod_{a \in \operatorname{Gen}(T)} x_{a}^{u(a)}$ is not in $J_{T}$ if and only if there exists $F \in \Pi_{T}$ such that $\operatorname{supp}\left(x^{u}\right) \subseteq F$. Here $\operatorname{supp}\left(x^{u}\right)$ is the support of $x^{u}$, that is, the set of elements $a \in \operatorname{Gen}(T)$ with $u(a) \neq 0$. Let $I_{F} \subset K[\operatorname{Gen}(T)]$ denote the ideal generated by the image of $\mathcal{I}_{F} \subset K[F]$ under the inclusion $K[F] \subseteq K[\operatorname{Gen}(T)]$ for $F \in \Pi_{T}$ and let $I_{T}=\sum_{F \in \Pi_{T}} I_{F}+J_{T}$. As observed in [10, Proposition 4.8] the face ring $K[T]=K[\operatorname{Gen}(T)] / I_{T}$ of the embedded toric complex $T$ agrees with the face ring of the rational fan $\left\{\operatorname{cone}(F): F \in \Pi_{T}\right\}$ considered in [21, Section 4] in the case where $M_{F}=\operatorname{cone}(F) \cap \mathbb{Z}^{d}$ for every $F \in \Pi_{T}$. Restricted to embedded toric complexes, the statement of our main result in Section 5 is:

Theorem 1.1 If $\operatorname{Gen}\left(\operatorname{sd}_{\omega T}\right)=\operatorname{Gen}(T)$ and $\cup_{F \in \Pi_{T}} \operatorname{cone}(F)=\cup_{G \in \Pi_{\mathrm{sd}_{\omega} T}} \operatorname{cone}(G)$, then the radical ideal $\operatorname{rad}\left(\operatorname{in}_{\omega}\left(I_{T}\right)\right)$ of $\operatorname{in}_{\omega}\left(I_{T}\right)$ agrees with the ideal $I_{\mathrm{sd}_{\omega T}}$.

The face ring $K[T]$ of an arbitrary toric complex $T$, as defined in 4.1, is a $K$-algebra of the form $K[X] / I_{T}$ where $K[X]$ is a polynomial ring and $I_{T}$ is a binomial ideal, that is, an ideal generated by binomials of the form $x^{u}-x^{v}$ and by monomials $x^{u}$. In the case where $K$ is a field every binomial ideal $I$ in $K[X]$ gives rise to a toric complex $T(I)$. In general 
it will not be possible to represent the toric complex $T(I)$ as an embedded toric complex. Theorem 4.4 contains a precise criterion on $I$ classifying the ideals for which the face ring $K[T(I)]$ is isomorphic to $K[X] / I$.

Simplicial toric complexes as defined in 3.4 correspond to abstract simplicial complexes in a strong sense. In particular, there is an abstract simplicial complex associated to a simplicial toric complex, and there is a simplicial toric complex associated to every abstract simplicial complex. The face ring of a simplicial toric complex agrees with the Stanley-Reisner ring of the associated abstract simplicial complex. Thus the ideals associated to simplicial toric complexes are monomial, so on the level of ideals, simplicial toric complexes correspond to the binomial ideals that happen to be monomial. It is hence very restrictive to require a toric complex to be simplicial.

In order to subdivide a toric complex $T$ we must have enough generators to be able to obtain additional faces. If $T$ is simplicial, there are not enough generators to do this. The solution to this problem is to add generators in a controlled way. In 4.9 we present a process of multiplying a toric complex by a natural number, and for $r \geq 2$ the multiple $r T$ of a toric complex $T$ has enough generators to possesses subdivisions. On the level of face rings, multiples of toric complexes roughly correspond to Veronese subrings of graded $K$-algebras, and their subdivisions correspond to deformations of the Veronese ring. The underlying fan of a multiple $r T$ of an embedded toric complex $T$ agrees with the underlying fan of $T$ itself, and the underlying fan of a subdivision of $r T$ is a subdivision of the underlying fan of $T$.

For the simplicial toric complex $T(\Delta)$ constructed from an abstract simplicial complex $\Delta$ (see Example 3.3) we define a particularly nice regular subdivision $\operatorname{esd}_{r}(T(\Delta))=$ $\operatorname{sd}_{\omega}(r T(\Delta))$ of $r T(\Delta)$. The toric complex esd $r(T(\Delta))$ is simplicial and the associated abstract simplicial complex is the $r$-fold edgewise subdivision $\operatorname{esd}_{r}(\Delta)$ of $\Delta$ defined in 6.1 . We show that the initial ideal $\operatorname{in}_{\omega}\left(I_{r T(\Delta)}\right)$ of the defining ideal $I_{r T(\Delta)}$ of the face ring of $r T(\Delta)$ is generated by square-free monomials, and we specify a Gröbner basis for $I_{r T(\Delta)}$ with respect to any monomial order $<$ refining the weight order $\omega$.

Some ideas presented in this paper can be found elsewhere in different contexts. First of all the concept of a toric complex is a variation on the polyhedral complexes defined in [15], and regular subdivision appears there under the name projective subdivision. There is a natural toric complex associated to simplicial fan, and this toric complex plays an important rôle in the work of Cox on the homogeneous coordinate ring of a toric variety [7]. The work of Cox has motivated the concept of stacky fans, corresponding to a kind of toric complexes consisting of subsets $F$ of a finitely generated abelian group instead of subsets of $\mathbb{Z}^{d}$, considered in [3]. The idea of considering arbitrary finite subsets $F$ of $\mathbb{Z}^{d}$ was promoted by Sturmfels and his coauthors, for example in [14] and [23]. They occasionally call $F$ a vector configuration. In [22] and [23] Sturmfels identified the radical of the initial ideal $\operatorname{in}_{\omega}\left(\mathcal{I}_{F}\right)$ of $\mathcal{I}_{F}$ with the defining ideal of a Stanley-Reisner ring in the case where $\omega$ defines a triangulation of cone $(F)$ using Gröbner bases and integer programming methods. We make a different approach using direct geometric arguments that allows us to extend Sturmfels' theorem to subdivisions of toric complexes and face rings over commutative rings instead of over fields. The results of Section 4 where we examine the face ring of a toric complex are motivated by the paper [10] of Eisenbud and Sturmfels. Finally, edgewise subdivision has been studied by many people. The basic idea is to subdivide a triangle along its edges: 


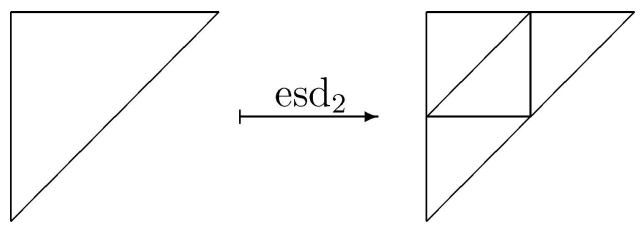

As observed by Freudenthal in [11], as opposed to barycentric subdivision, the pieces of iterated edgewise subdivision do not become long and thin. Knudsen and Mumford studied variations of edgewise subdivision of polyhedral complexes in [15]. The name edgewise subdivision was introduced by Grayson in [13] where edgewise subdivision of simplicial sets is used to obtain operations in higher algebraic $K$-theory. Edgewise subdivision of cyclic sets is essential for the construction of topological cyclic homology [2].

The paper is organized as follows: in Section 3 we give our definition of a toric complex together with some examples, and we characterize elementary properties of toric complexes. In Section 4 we define the face ring of a toric complex, and in the case where $K$ is a field we characterize the $K$-algebras occurring as face-rings. Furthermore we show that the face ring is compatible with gluing of toric complexes and we give a construction on toric complexes corresponding to the Veronese subring of a graded ring. In Section 5 we introduce regular subdivisions of toric complexes and we prove our main result Theorem 5.11. In Section 6 we discuss a particular regular subdivision of the toric complex associated to an abstract simplicial complex, which we call the edgewise subdivision.

The authors are grateful to Prof. W. Bruns for inspiring discussions on the subject of the paper.

\section{Prerequisites}

In this section we fix some notation and recall some standard definitions. Let $F$ be a finite subset of $\mathbb{R}^{d}$. A convex combination of elements of $F$ is a sum $\sum_{a \in F} r_{a} a$ with $0 \leq r_{a}$ for $a \in F$ and $\sum_{a \in F} r_{a}=1$. The set of convex combinations $\operatorname{conv}(F)$ of elements of $F$ is called the convex hull of $F$. Similarly, a positive linear combination of elements of $F$ is a sum $\sum_{a \in F}^{n} r_{a} a$ with $r_{a} \in \mathbb{R}_{+}=\{x \in \mathbb{R}: x \geq 0\}$ for $a \in F$. The set cone $(F)$ of positive linear combinations of elements of $F$ is called the cone generated by $F$. By convention cone $(\emptyset)=\{0\}$ and $\operatorname{conv}(\emptyset)=\emptyset$.

To a linear form $\alpha$ on $\mathbb{R}^{d}$ and $c \in \mathbb{R}$ we associate the affine hyperplane $H_{\alpha}(c)=\alpha^{-1}(c)$ and the half-space $H_{\alpha}^{-}(c)=\alpha^{-1}((-\infty, c])$. An intersection $P=\bigcap_{i=1}^{n} H_{\alpha_{i}}^{-}\left(c_{i}\right)$ of finitely many half-spaces is called a polyhedron. A face of a polyhedron $P$ is the intersection of $P$ with an additional hyperplane $H_{\beta}(d)$ with the property that $P \subseteq H_{\beta}^{-}(d)$. A polytope is a bounded polyhedron. The set $\operatorname{conv}(F)$ is a polytope for every finite subset $F$ of $\mathbb{R}^{d}$ and every polytope is of this form. The polytope $\operatorname{conv}(F)$ is a simplex if every element in $\operatorname{conv}(F)$ has a unique representation as a convex combination of the elements of $F$. A cone is a finite intersection of half-spaces of the form $H_{\alpha_{i}}^{-}(0)$. The set cone $(F)$ is a cone for every finite subset $F$ of $\mathbb{R}^{d}$ and every cone is of this form. For the theory 
of polyhedrons and related things we refer to the books of Schrijver [18] and Ziegler [24].

Given a commutative monoid $M$, the monoid algebra $K[M]$ is the set of functions $f: M \rightarrow K$ with finite support with $(f+g)(m)=f(m)+g(m)$ and $(f g)(m)=\sum_{m_{1}+m_{2}=m}$ $\times f\left(m_{1}\right) g\left(m_{2}\right)$ for $m \in M$. Given a finite set $F$, the free commutative monoid on $F$ is the monoid $\mathbb{N}^{F}$ consisting of functions $u: F \rightarrow \mathbb{N}$, and with $(u+v)(a)=u(a)+v(a)$ for $u, v \in \mathbb{N}^{F}$ and $a \in F$. The polynomial ring $K[F]$ is the monoid algebra $K\left[\mathbb{N}^{F}\right]$. A monomial in $K[F]$ is a function of the form $x^{u}: \mathbb{N}^{F} \rightarrow K$ with $x^{u}(u)=1$ and $x^{u}(v)=0$ for $v \neq u$, and a polynomial $f \in K[F]$ can be represented as the sum $f=\sum_{u \in \mathbb{N}^{F}} f_{u} x^{u}$ where $f_{u}=f(u) \in K$. For $a \in F$ we let $u_{a}: F \rightarrow \mathbb{N}$ denote the function defined by $u_{a}(a)=1$ and $u_{a}(b)=0$ for $b \neq a$ and we let $x_{a}=x^{u_{a}}$. If $G \subseteq F$, then we let $x_{G}$ be the square-free monomial $\prod_{a \in G} x_{a}$.

Given a function $f: F \rightarrow G$ of finite sets, the homomorphism $f_{*}: K[F] \rightarrow K[G]$ is defined by $f_{*}\left(x_{a}\right)=x_{f(a)}$ for $x_{a} \in K[F]$, and the homomorphism $f^{*}: K[G] \rightarrow K[F]$ is defined by $f^{*}\left(x_{b}\right)=\sum_{f(a)=b} x_{a}$ for $x_{b} \in K[G]$. Here a sum indexed on the empty set is zero by convention. The $r$-th Veronese subring of a $\mathbb{Z}$-graded ring $R=\oplus_{i \in \mathbb{Z}} R_{i}$ is the $\mathbb{Z}$-graded ring $R^{(r)}=\oplus_{i \in \mathbb{Z}} R_{r i}$.

\section{Toric complexes}

In this section we introduce toric complexes, the objects studied in this paper.

Definition 3.1 A toric complex $T$ consists of:

(a) A finite partially ordered set $\left(\Pi_{T}, \subseteq\right)$ consisting of finite sets, ordered by inclusion,

(b) for every $F \in \Pi_{T}$ an injective function $T_{F}: F \rightarrow \mathbb{Z}^{d_{F}} \backslash\{0\}$ for some $d_{F}>0$,

(c) for every pair $F, G \in \Pi_{T}$ with $F \subseteq G$ an injection $T_{F G}: \mathbb{Z}^{d_{F}} \rightarrow \mathbb{Z}^{d_{G}}$ of abelian groups,

subject to the following conditions:

(i) if $F, G \in \Pi_{T}$, then $F \cap G \in \Pi_{T}$,

(ii) for all $F, G \in \Pi_{T}$ with $F \subseteq G$ and $a \in F$ we have $T_{F G}\left(T_{F}(a)\right)=T_{G}(a)$,

(iii) for every triple $F, G, H \in \Pi_{T}$ with $F \subseteq G \subseteq H$ we have $T_{G H} \circ T_{F G}=T_{F H}$,

(iv) if $G \in \Pi_{T}$ and $F \subseteq G$, then $F \in \Pi_{T}$ if and only if cone( $\left.T_{G}(F)\right)$ is a face of cone $\left(T_{G}(G)\right)$ satisfying $T_{G}(F)=\operatorname{cone}\left(T_{G}(F)\right) \cap T_{G}(G)$.

The set of generators of $T$ is the union $\operatorname{Gen}(T)=\cup_{F \in \Pi_{T}} F$ and the faces of $T$ are the elements $F \in \Pi_{T}$. If $F$ and $G$ are faces of $T$ with $F \subseteq G$ we say that $F$ is a face of $G$ in $T$. For a face $F \in \Pi_{T}$ we define the monoid homomorphism $T_{F}: \mathbb{N}^{F} \rightarrow \mathbb{Z}^{d_{F}}$, $T_{F}(u)=\sum_{a \in F} u(a) T_{F}(a)$.

A toric complex $T$ is embedded if there exist $d \in \mathbb{N}$ such that $T_{F G}$ is the identity on $\mathbb{R}^{d}$ for every $F, G \in \Pi_{T}$ with $F \subseteq G$. In particular, we have $T_{F}(a)=T_{G}(a)$ for $a \in F$. Note 
that for a toric complex $T$ the collection $\left\{\operatorname{cone}(F): F \in \Pi_{T}\right\}$ is a polyhedral complex as considered for example in [15] consisting of rational cones. If $T$ is embedded, then this is a fan in the lattice $\mathbb{Z}^{d}$.

Whenever appropriate we shall consider a partially ordered set $\Pi$ as a category with objects set equal to the underlying set of $\Pi$ and with morphism sets $\Pi(F, G)$ consisting of exactly one element $F \rightarrow G$ if $F \leq G$ and with $\Pi(F, G)$ empty otherwise. We let $\Pi^{\text {op }}$ denote the opposite category of $\Pi$ with the same set of objects as $\Pi$ and with $\Pi^{\mathrm{op}}(G, F)=$ $\Pi(F, G)$.

Given a toric complex $T$ and elements $F \subseteq G$ of $\Pi_{T}$, there are continuous injective maps $\operatorname{conv}\left(T_{F}(F)\right) \rightarrow \operatorname{conv}\left(T_{G}(G)\right)$ and cone $\left(T_{F}(F)\right) \rightarrow \operatorname{cone}\left(T_{G}(G)\right)$ induced by the injective homomorphism $T_{F G}$. Thus we can consider $F \mapsto \operatorname{conv}\left(T_{F}(F)\right)$ and $F \mapsto \operatorname{cone}\left(T_{F}(F)\right)$ as functors from $\Pi_{T}$ to the category of topological spaces. We define the spaces $|T|$ and $\|T\|$ as the colimits:

$$
|T|=\underset{F \in \Pi_{T}}{\lim } \operatorname{conv}\left(T_{F}(F)\right) \text { and }\|T\|=\underset{F \in \Pi_{T}}{\lim } \operatorname{cone}\left(T_{F}(F)\right)
$$

Observe that if $T$ is embedded, then the topological spaces $|T|=\bigcup_{F \in \Pi_{T}} \operatorname{conv}\left(T_{F}(F)\right)$ and $\|T\|=\bigcup_{F \in \Pi_{T}}$ cone $\left(T_{F}(F)\right)$ are unions of subspaces of $\mathbb{R}^{d}$.

Example 3.2 A subset $G$ of $\mathbb{Z}^{d}$ gives rise to an embedded toric complex $T(G)$. The function $T(G)_{G}$ is the inclusion of $G$ in $\mathbb{Z}^{d}$ and $\Pi_{T(G)}$ consist of those subsets $F$ of $G$ satisfying axiom (iv) of Definition 3.1.

This type of toric complexes correspond to vector configurations as studied for example in [14] or [19].

Example 3.3 Let $\Delta$ be an abstract simplicial complex on the vertex set $V=\{1, \ldots, d+1\}$, i.e. $\Delta$ is a set of subsets of $V$ and $F \subseteq G \in \Delta$ implies $F \in \Delta$. Let $e_{1}, \ldots, e_{d+1}$ denote the standard generators of $\mathbb{Z}^{d+1}$ and define $\iota_{V}: V \rightarrow \mathbb{Z}^{d+1}$ by $\iota_{V}(i)=e_{i}+e_{i+1}+\cdots+e_{d+1}$ for $1 \leq i \leq d+1$. We associate an embedded toric complex $T(\Delta)$ to $\Delta$ as follows:

(a) $\Pi_{T(\Delta)}$ is the partially ordered set $\Delta$,

(b) for $F \in \Delta$ we let $T(\Delta)_{F}$ denote the restriction of $\iota_{V}$ to $F \subseteq V$,

Observe that $|T(\Delta)|$ is homeomorphic to the usual geometric realization of $\Delta$.

Definition 3.4 Let $T$ be a toric complex.

(i) $T$ is $\mathbb{Z}$-graded if $T_{F G}: \mathbb{Z}^{d_{F}} \rightarrow \mathbb{Z}^{d_{G}}$ preserves the last coordinate for all $F, G \in \Pi_{T}$ with $F \subseteq G$.

(ii) $T$ is $\mathbb{N}$-graded if it is $\mathbb{Z}$-graded and the last coordinate of $T_{F}(a)$ is positive for all $a \in F$ and $F \in \Pi_{T}$. 
(iii) $T$ is standard graded if it is $\mathbb{Z}$-graded and the last coordinate of $T_{F}(a)$ is 1 for all $a \in F$ and $F \in \Pi_{T}$.

(iv) $T$ is pointed if $\emptyset \in \Pi_{F}$.

(v) $T$ is simplicial if $\Pi_{T}$ is an abstract simplicial complex.

Observe that the toric complex $T(\Delta)$ of Example 3.3 is simplicial.

Example 3.5 Let $\Sigma$ be a rational fan in a lattice $N$, that is, $\Sigma$ is a finite collection of rational polyhedral cones $\sigma$ in $N_{\mathbb{R}}=N \otimes_{\mathbb{Z}} \mathbb{R}$, satisfying: every face of a cone in $\Sigma$ is also a cone in $\Sigma$, and the intersection of two cones in $\Sigma$ is a face of each. Choosing a set $G_{\sigma} \subseteq N \backslash\{0\}$ of generators for each cone $\sigma$ in $\Sigma$ and an isomorphism $\varphi: N \rightarrow \mathbb{Z}^{d}$ we can construct an embedded toric complex $T\left(\Sigma,\left(G_{\sigma}\right)_{\sigma \in \Sigma}, \varphi\right)$ as follows: for $\sigma \in \Sigma$ we let $F_{\sigma}$ denote the union of the sets $\varphi\left(G_{\gamma}\right)$ of images of generators of faces $\gamma$ of $\sigma$ under $\varphi$ and we define $\prod_{T\left(\Sigma,\left(G_{\sigma}\right)_{\sigma \in \Sigma}, \varphi\right)}$ to be the collection of subsets of $\mathbb{Z}^{d}$ of the form $F_{\sigma}$ for $\sigma \in \Sigma$.

There is a preferred toric complex associated to a simplicial fan, namely the one where we take $G_{\rho}$ to be the set of unique generators $\rho \cap N$ if $\rho$ is a ray in $\Sigma$, and where $G_{\sigma}$ is the union over the rays $\rho$ in $\sigma$ of the sets $G_{\rho}$. On the other hand, the underlying fan \{cone $\left(T_{F}(F)\right): F \in \Pi_{T}$ \} of an embedded toric complex $T$ is simplicial if $T$ is simplicial. If we want to subdivide $T\left(\Sigma,\left(G_{\sigma}\right)_{\sigma \in \Sigma}, \varphi\right)$, it is necessary to choose the sets $G_{\sigma}$ different from the preferred ones described above. In Definition 4.9 below we present a way to do this.

Example 3.6 Lattice polyhedral complexes in the sense of [5, Definition 2.1] correspond to the subclass of the class of standard graded toric complexes consisting of toric complexes $T$ with the property that $F$ is the set of vertices of the polytope $\operatorname{conv}\left(T_{F}(F)\right)$ for every $F \in \Pi_{T}$. For a concrete example of a non-embeddable toric complex we refer to [5, Proposition 2.3].

Lemma 3.7 Let $T$ be a toric complex.

(i) $T$ is pointed if and only if zero is a vertex of cone $\left(T_{F}(F)\right)$ for every $F \in \Pi_{T}$.

(ii) If $T$ is $\mathbb{N}$-graded, then it is pointed.

(iii) $T$ is simplicial if and only if for all $F \in \Pi_{T}$ the elements $T_{F}$ (a) for $a \in F$ are linearly independent.

Proof: We only prove (iii). Assume that for all $G \in \Pi_{T}$ the elements $T_{G}(a)$ for $a \in G$ are linearly independent. If $F \subseteq G$ and $G \in \Pi_{T}$, then it is easy to see that cone $\left(T_{G}(F)\right)$ is a face of cone $\left(T_{G}(G)\right)$ and $T_{G}(\bar{F})=T_{G}(G) \cap \operatorname{cone}\left(T_{G}(F)\right)$. Since $T$ is a toric complex this implies $F \in \Pi_{T}$ and thus $\Pi_{T}$ is a simplicial complex.

On the other hand, suppose that $\Pi_{T}$ is an abstract simplicial complex and let $G \in \Pi_{T}$. Assume that the elements of $T_{G}(G)$ are linearly dependent. Thus for some $a \in G$ there is a relation $T_{G}(a)=\sum_{b \in G, b \neq a} \lambda_{b} T_{G}(b)$ with $\lambda_{b} \in \mathbb{R}$. By our assumption $F=G \backslash\{a\}$ generates a proper face cone $\left(T_{G}(F)\right)$ of cone $\left(T_{G}(G)\right)$ with $T_{G}(F)=\operatorname{cone}\left(T_{G}(F)\right) \cap T_{G}(G)$. Choosing a linear form $\alpha$ on $\mathbb{R}^{d_{G}}$ with cone $\left(T_{G}(F)\right)=\alpha^{-1}(0) \cap \operatorname{cone}\left(T_{G}(G)\right)$ we get the contradiction $0 \neq \alpha\left(T_{G}(a)\right)=\sum_{b \in F} \lambda_{b} \alpha\left(T_{G}(b)\right)=0$. Hence the elements in $T_{G}(G)$ are linearly independent. 
Definition 3.8 We call $S$ a subcomplex of a toric complex $T$ and write $S \subseteq T$ if $S$ is a toric complex with $\Pi_{S} \subseteq \Pi_{T}$ such that $S_{F}=T_{F}$ and $S_{F G}=T_{F G}$ for $F, G \in \Pi_{S}$ with $F \subseteq G$.

Observe that by the definition of a toric complex we have for $G \in \Pi_{S}$ and $F \subseteq G$ that $F \in \Pi_{S}$ if and only if $F \in \Pi_{T}$.

\section{The face ring of a toric complex}

Stanley associated in [21] a $K$-algebra to a rational fan. In this section we translate Stanley's definition to the situation of toric complexes. Recall the notation introduced in Sections 1 and 2 which will be used in the following.

Definition 4.1 Let $T$ be a toric complex. The face ring $K[T]$ is the $K$-algebra $K[T]=$ $K[\operatorname{Gen}(T)] / I_{T}$, where $I_{T}=J_{T}+\sum_{F \in \Pi_{T}} I_{F}$. Here $J_{T}$ is the monomial ideal such that a monomial $x^{u}$ is not in $J_{T}$ if and only if there exists $F \in \Pi_{T}$ with $\operatorname{supp}\left(x^{u}\right) \subseteq F$. For $F \in \Pi_{T}$ the ideal $I_{F}$ is generated by the image of the kernel $\mathcal{I}_{F} \subseteq K[F]$ of the homomorphism $K[F] \rightarrow K\left[\mathbb{Z}^{d_{F}}\right]$ induced by $T_{F}$ under the inclusion $K[F] \rightarrow K[\operatorname{Gen}(T)]$.

Recall that an ideal in a polynomial ring over $K$ is binomial, if it is generated by binomials $x^{u}-x^{v}$ and monomials $x^{u}$. Such binomial ideals are strict in the sense that we do not allow the generators to have coefficients different from 1. A monomial ideal is an ideal generated by monomials.

Observe that the ideal $I_{T}$ of a toric complex is binomial, because for every $F \in \Pi_{T}$ the ideal $I_{F}$ is generated by the binomials $x^{u}-x^{v}$ where $u, v: F \rightarrow \mathbb{N}$ satisfy that $T_{F}(u)=$ $T_{F}(v)$ (e.g. see [23, Lemma 4.1]). Note that if $I_{F}$ is a monomial ideal, then $I_{F}=0$. If $T$ is $\mathbb{Z}$-graded, then $K[T]$ inherits a $\mathbb{Z}$-grading, and $K[T]$ is $\mathbb{N}$-graded if $T$ is $\mathbb{N}$-graded. If $T$ is standard graded, then $K[T]$ is generated by elements of degree one.

Theorem 4.2 Let $S$ be a subcomplex of a toric complex $T$ and let $i: \operatorname{Gen}(S) \rightarrow \operatorname{Gen}(T)$ denote the inclusion of the generators of $S$ in the generators of $T$.

(i) The homomorphism $i^{*}: K[\operatorname{Gen}(T)] \rightarrow K[\operatorname{Gen}(S)]$ induces a surjective homomorphism $i_{S T}^{*}: K[T] \rightarrow K[S]$ with

$$
\operatorname{Ker}\left(i_{S T}^{*}\right)=\left(x_{F}: F \subseteq \operatorname{Gen}(T) \text { is not contained in any } G \in \Pi_{S}\right) .
$$

(ii) The homomorphism $i_{*}: K[\operatorname{Gen}(S)] \rightarrow K[\operatorname{Gen}(T)]$ induces an injective homomorphism $i_{S T *}: K[S] \rightarrow K[T]$ with $i_{S T}^{*} \circ i_{S T *}=\mathrm{id}_{K[S]}$.

Proof: To prove that $i_{S T}^{*}$ is well defined we need to check that $i^{*}$ maps the ideal $I_{T}=$ $J_{T}+\sum_{F \in \Pi_{T}} I_{F}$ into the ideal $I_{S}=J_{S}+\sum_{G \in \Pi_{S}} I_{G}$.

Clearly $J_{T}$ is mapped into $J_{S}$. For $F \in \Pi_{T}$ the ideal $I_{F}$ is generated by binomials $x^{u}-x^{v}$ where $x^{u}$ and $x^{v}$ have support contained in $F$ and satisfy that $T_{F}(u)=T_{F}(v)$. For $G \subseteq F$ such that cone $\left(T_{F}(G)\right)$ is a face of $\operatorname{cone}\left(T_{F}(F)\right)$ we have $\operatorname{supp}\left(x^{u}\right) \subseteq G$ if and only if $T_{F}(u) \in \operatorname{cone}\left(T_{F}(G)\right)$. Thus $\operatorname{supp}\left(x^{u}\right) \subseteq G$ if and only if $\operatorname{supp}\left(x^{v}\right) \subseteq G$. The element 
$i^{*}\left(x^{u}\right)$ is not in $J_{S}$ if and only if there exists a $G \in \Pi_{S}$ such that $\operatorname{supp}\left(x^{u}\right) \subseteq G$. Hence $i^{*}\left(x^{u}\right)$ is not in $J_{S}$ if and only if the same is the case for $i^{*}\left(x^{v}\right)$. Now if $i^{*}\left(x^{u}\right)$ and $i^{*}\left(x^{v}\right)$ are in $J_{S}$ we are done. Otherwise there exists $G \in \Pi_{S} \operatorname{such}$ that $\operatorname{supp}\left(x^{u}\right)$ and $\operatorname{supp}\left(x^{v}\right)$ are contained in $G$ and therefore $i^{*}\left(x^{u}-x^{v}\right)$ is in $I_{G}$. We conclude that $i^{*}\left(I_{T}\right) \subseteq I_{S}$ and we get the induced homomorphism $i_{S T}^{*}: K[T] \rightarrow K[S]$. This homomorphism is clearly surjective and has the described kernel.

Analogously one shows that $i_{S T_{*}}: K[S] \rightarrow K[T]$ is an injective homomorphism with $i_{S T}^{*} \circ i_{S T *}=\mathrm{id}_{K[S]}$. This concludes the proof.

We need the following variation of Corollary 2.4 in [10].

Lemma 4.3 Let $K$ be a field and let $P$ be a prime ideal in $K[F]$ generated by binomials $x^{u}-x^{v}$ for some finite set $F$. There exists a unique direct summand $L_{P}$ of $\mathbb{Z}^{F}$ such that the composition $K[F]=K\left[\mathbb{N}^{F}\right] \subseteq K\left[\mathbb{Z}^{F}\right] \rightarrow K\left[\mathbb{Z}^{F} / L_{P}\right]$ induces an embedding $K[F] / P \subseteq K\left[\mathbb{Z}^{F} / L_{P}\right]$.

Proof: Since $x^{m+n}-1=x^{m}\left(x^{n}-1\right)+\left(x^{m}-1\right)$ the elements $m \in \mathbb{Z}^{F}$ satisfying $x^{m}-1 \in P K\left[\mathbb{Z}^{F}\right]$ form a lattice $L_{P}$ with $P K\left[\mathbb{Z}^{F}\right]$ contained in the kernel $I\left(L_{P}\right)$ of the homomorphism $\gamma: K\left[\mathbb{Z}^{F}\right] \rightarrow K\left[\mathbb{Z}^{F} / L_{P}\right]$. Since $P=P K\left[\mathbb{Z}^{F}\right] \cap K\left[\mathbb{N}^{F}\right]$, it suffices to prove that the homomorphism $\delta: K\left[\mathbb{Z}^{F}\right] / P K\left[\mathbb{Z}^{F}\right] \rightarrow K\left[\mathbb{Z}^{F} / L_{P}\right]$ is an isomorphism and that $L_{P}$ is a direct summand of $\mathbb{Z}^{F}$. If $\delta$ is an isomorphism, then $L_{P}$ must be a direct summand of $\mathbb{Z}^{F}$ because $P K\left[\mathbb{Z}^{F}\right]$ is a prime ideal.

An element $f=\sum_{u \in \mathbb{Z}^{F}} f_{u} x^{u}$ is mapped to

$$
\gamma(f)=\sum_{v+L_{P} \in \mathbb{Z}^{F} / L_{P}}\left(\sum_{m \in L_{P}} f_{v+m}\right) x^{v+L_{P}} .
$$

We have that $\gamma(f)=0$ if and only if $\sum_{m \in L_{P}} f_{v+m}=0$ for all $v+L_{P} \in \mathbb{Z}^{F} / L_{P}$ and this implies that $\sum_{m \in L_{P}} f_{v+m} x^{m}$ is in the ideal generated by the $x^{m}-1$. Thus we see that the ideal $I\left(L_{P}\right)$ is generated by the elements $x^{m}-1$ for $m \in L_{P}$. We conclude that $I\left(L_{P}\right) \subseteq P K\left[\mathbb{Z}^{F}\right]$, and that the homomorphism $\delta$ in question is an isomorphism. This concludes the proof.

Let $I \subset K\left[x_{1}, \ldots, x_{n}\right]$ be a binomial ideal. For a subset $F \subseteq\{1, \ldots, n\}$ let $P_{F}=$ $I \cap K[F]$. As in [10, Cor. 1.3] we see that $P_{F}$ is a binomial ideal in $K[F]$. Let $\Pi_{T(I)}$ denote the collection of subsets $F$ of $\{1, \ldots, n\}$ such that $P_{F}$ is a prime ideal not containing any monomial and such that the projection $K\left[x_{1}, \ldots, x_{n}\right] \rightarrow K[F]$ induces a homomorphism $K\left[x_{1}, \ldots, x_{n}\right] / I \rightarrow K[F] / P_{F}$.

Theorem 4.4 Let $K$ be a field, $X=\{1, \ldots, n\}$ and $I \subset K[X]=K\left[x_{1}, \ldots, x_{n}\right]$ be a binomial ideal. There exists a toric complex $T(I)$ with face set $\Pi_{T(I)}$ and $K[T(I)] \cong$ $K[X] / \bigcap_{F \in \Pi_{T(I)}}\left(P_{F}+\left(x_{i}: i \notin F\right)\right)$. In particular, $I=\bigcap_{F \in \Pi_{T(I)}}\left(P_{F}+\left(x_{i}: i \notin F\right)\right)$ if and only if the natural homomorphism $K[X] / I \rightarrow K[T(I)]$ is an isomorphism. 
Proof: Let us first construct an abstract toric complex $T^{\prime}(I)$ in the sense that the targets for the maps $T_{F}^{\prime}$ for $F \in \Pi_{T^{\prime}(I)}=\Pi_{T(I)}$ are abstract finitely generated free abelian groups instead of abelian groups of the form $\mathbb{Z}^{d_{F}}$. For every $F \in \Pi_{T(I)}$ there is an isomorphism $K[X] / I+\left(x_{i}: i \notin F\right) \cong K[F] / P_{F}$. Let $L_{P_{F}}$ be as in Lemma 4.3 and let $T_{F}^{\prime}$ denote the composition $F \subseteq \mathbb{N}^{F} \subseteq \mathbb{Z}^{F} \rightarrow \mathbb{Z}^{F} / L_{P_{F}}$. If $F \subseteq G$, then the inclusion $\mathbb{Z}^{F} \subseteq \mathbb{Z}^{G}$ and the fact $P_{F}=P_{G} \cap K[F]$ induce an inclusion $T_{F G}^{\prime}: \mathbb{Z}^{F} / L_{P_{F}} \rightarrow \mathbb{Z}^{G} / L_{P_{G}}$. Choosing isomorphisms $\mathbb{Z}^{F} / L_{P_{F}} \cong \mathbb{Z}^{d_{F}}$ and applying [6, Theorem 6.1.7] it is straightforward to check that we obtain a toric complex $T(I)$.

In order to identify $K[T(I)]$ we assume without loss of generality that $\operatorname{Gen}(T(I))=X$. Note that for $F \in \Pi_{T(I)}$ the ideal $P_{F}$ agrees with the kernel $\mathcal{I}_{F}$ of the homomorphism $K[F] \rightarrow K\left[\mathbb{Z}^{d_{F}}\right]$ induced by $T_{F}$. Considering the inclusions of toric complexes $T(F) \subseteq$ $T(I)$, Theorem 4.2 ensures that $i_{*} i^{*}(f) \in I_{F}$ for $f \in I_{T(I)}$. Since $f-i_{*} i^{*}(f) \in\left(x_{i}: i \notin F\right)$ and $f$ was arbitrary, we get that $I_{T(I)}$ is contained in $\left(P_{F}+\left(x_{i}: i \notin F\right)\right)$. Thus $I_{T(I)}$ is contained in $\bigcap_{F \in \Pi_{T(I)}}\left(P_{F}+\left(x_{i}: i \notin F\right)\right)$.

Let $f \in \bigcap_{F \in \Pi_{T(I)}}\left(P_{F}+\left(x_{i}: i \notin F\right)\right)$ and let $F_{1}, \ldots, F_{m}$ be the maximal faces of $T(I)$. Recall that the inclusion $i$ of $F_{1}$ in $X$ induces homomorphisms $i^{*}: K[X] \rightarrow K\left[F_{1}\right]$ and $i_{*}: K\left[F_{1}\right] \rightarrow K[X]$. The element $f-i_{*} i^{*}(f) \in K[X]$ is in the ideal $\left(\bigcap_{F \in \Pi_{T(I)}}\left(P_{F}+\left(x_{i}: i \notin\right.\right.\right.$ $F))) \cap\left(x_{i}: i \notin F_{1}\right)$ because $i_{*} i^{*}(f) \in I_{F} \subseteq I_{T(I)}$. Proceeding by induction on $m$ we find an element $g$ of $I_{T(I)}$ such that $f-g \in \bigcap_{1 \leq j \leq m}\left(x_{i}: i \notin F_{j}\right)=J_{T(I)} \subseteq I_{T(I)}$. Hence $f \in I_{T(I)}$ and it follows that $I_{T(I)}=\bigcap_{F \in \Pi_{T(I)}}\left(P_{F}+\left(x_{i}: i \notin F\right)\right)$ and that $K[T(I)]=$ $K[X] / \bigcap_{F \in \Pi_{T(I)}}\left(P_{F}+\left(x_{i}: i \notin F\right)\right)$. The last claim of the theorem is a consequence of this fact.

If a toric complex $T$ is simplicial, then all the ideals $I_{F}=0$ for $F \in \Pi_{T}$ because the linear independence of the elements of $T_{F}(F)$ implies that the ring $K\left[M_{T_{F}(F)}\right]$ is a polynomial ring. Hence $I_{T}=J_{T}$ is a square-free monomial ideal and the face $\operatorname{ring} K[T]$ coincides with the so-called Stanley-Reisner ring of the abstract simplicial complex $\Pi_{T}$. (See for example [20] for more details on this subject.) We show that the converse is also true.

Proposition 4.5 A toric complex $T$ is simplicial if and only if $I_{T}$ is a monomial ideal. In this case $K[T]$ is the Stanley-Reisner ring of the abstract simplicial complex $\Pi_{T}$.

Proof: If a toric complex $T$ is simplicial, then $I_{T}$ is a monomial ideal as shown above.

Therefore assume that $I_{T}$ is a monomial ideal. Given $F \in \Pi_{T}$ we consider the toric subcomplex $T(F)$ of Example 3.2. Let $i: F=\operatorname{Gen}(T(F)) \rightarrow \operatorname{Gen}(T)$ be the inclusion. Since $I_{T}$ is monomial, so is the ideal $I_{T(F)}=i^{*}\left(I_{T}\right)$. It follows that $I_{T(F)}=0$, because $K[F] / I_{T(F)}$ is an affine monoid ring. Thus the elements of $T_{F}(F)$ are linearly independent. By 3.7 (iii) we conclude that $T$ is simplicial. Then $I_{T}=J_{T}$ and $K[T]$ is the Stanley-Reisner ring of $\Pi_{T}$.

Given a toric complex $T$ and subcomplexes $Q, S \subset T$, there is a subcomplex $R=Q \cap S$ of $T$ defined by letting $\Pi_{R}=\Pi_{Q} \cap \Pi_{S}$. One can show that the space $|R|=|Q \cap S|$ is isomorphic to $|Q| \cap|S|$. We write $T=Q \cup S$ if $\Pi_{T}=\Pi_{Q} \cup \Pi_{S}$. Since there are 
homomorphisms $i_{R Q}^{*}: K[Q] \rightarrow K[R]$ and $i_{R S}^{*}: K[S] \rightarrow K[R]$ we can consider the fiber product $K[Q] \times_{K[R]} K[S]$.

Observe that the homomorphisms $i_{S T *}$ and $i_{S T}^{*}$ are twins in the sense of Notbohm and Ray [16], that is $i_{Q T}{ }^{*} i_{S T *}=i_{R Q_{*}} i_{R S}{ }^{*}$ and $i_{S T}{ }^{*} i_{Q T_{*}}=i_{R S *} i_{R Q}{ }^{*}$. The face ring of $T$ and the face rings of $Q$ and $S$ are related as follows:

Proposition 4.6 If $T=Q \cup S$, then the homomorphism

$$
\left(i_{Q T}^{*}, i_{S T}^{*}\right): K[T] \rightarrow K[Q] \times_{K[Q \cap S]} K[S]
$$

is an isomorphism.

Proof: As above let $R=S \cap Q$. We prove that the additive homomorphism $\beta: K[Q] \times_{K[R]}$ $K[S] \rightarrow K[T]$ defined by $\beta(a, b)=i_{Q T_{*}}(a)+i_{S T *}(b)-i_{R T *}\left(i_{R Q^{*}}(a)\right)$ for $(a, b) \in$ $K[Q] \times_{K[R]} K[S]$ is a homomorphism of rings. Using the fact that $i_{S T *}$ and $i_{S T}^{*}$ are twins we see that $\beta$ is an additive inverse to $\left(i_{Q T}^{*}, i_{S T}^{*}\right)$.

The only thing left to check is that $\beta(a, b) \beta\left(a^{\prime}, b^{\prime}\right)=\beta\left(a a^{\prime}, b b^{\prime}\right)$. Since the support $\operatorname{supp}\left(x^{u}\right)$ of a monomial $x^{u}$ in the polynomial $\left(i_{Q T_{*}}(a)-i_{R T *} i_{R Q}^{*}(a)\right)$ satisfies that $\operatorname{supp}\left(x^{u}\right) \cap$ $(\operatorname{Gen}(Q) \backslash \operatorname{Gen}(R)) \neq \emptyset$, and since $\operatorname{supp}\left(x^{v}\right) \cap(\operatorname{Gen}(S) \backslash \operatorname{Gen}(R)) \neq \emptyset$ for a monomial $x^{v}$ in the polynomial $\left(i_{S T *}\left(b^{\prime}\right)-i_{R T *} i_{R S}^{*}\left(b^{\prime}\right)\right)$ we have that

$$
\left(i_{Q T_{*}}(a)-i_{R T *} i_{R Q}^{*}(a)\right)\left(i_{S T *}\left(b^{\prime}\right)-i_{R T *} i_{R S}^{*}\left(b^{\prime}\right)\right) \in J_{T} .
$$

Similarly we see that $\left(i_{S T *}(b)-i_{R T *} i_{R S}^{*}(b)\right)\left(i_{Q T_{*}}\left(a^{\prime}\right)-i_{R T *} i_{R Q}^{*}\left(a^{\prime}\right)\right) \in J_{T}$, and we compute that

$$
\begin{aligned}
& \beta\left(a a^{\prime}, b b^{\prime}\right)=\beta\left(a a^{\prime}, b b^{\prime}\right)+\left(i_{Q T_{*}}(a)-i_{R T *} i_{R Q}^{*}(a)\right)\left(i_{S T *}\left(b^{\prime}\right)-i_{R T *} i_{R S}^{*}\left(b^{\prime}\right)\right) \\
& +\left(i_{S T *}(b)-i_{R T *} i_{R S}^{*}(b)\right)\left(i_{Q T_{*}}\left(a^{\prime}\right)-i_{R T *} i_{R Q}^{*}\left(a^{\prime}\right)\right) \\
& =i_{Q T_{*}}\left(a a^{\prime}\right)+i_{S T *}\left(b b^{\prime}\right)-i_{R T *} i_{R Q}^{*}\left(a a^{\prime}\right)+i_{Q T_{*}}(a) i_{S T *}\left(b^{\prime}\right) \\
& -i_{Q T_{*}}(a) i_{R T *} i_{R S}^{*}\left(b^{\prime}\right)-i_{R T *} i_{R Q}^{*}(a) i_{S T *}\left(b^{\prime}\right) \\
& +i_{R T *} i_{R Q}^{*}(a) i_{R T *} i_{R S}^{*}\left(b^{\prime}\right)+i_{S T *}(b) i_{Q T_{*}}\left(a^{\prime}\right)-i_{R T *} i_{R S}^{*}(b) i_{Q T_{*}}\left(a^{\prime}\right) \\
& -i_{S T *}(b) i_{R T *} i_{R Q}^{*}\left(a^{\prime}\right)+i_{R T *} i_{R S}^{*}(b) i_{R T *} i_{R Q}^{*}\left(a^{\prime}\right) \\
& =i_{Q T_{*}}(a) i_{Q T_{*}}\left(a^{\prime}\right)+i_{Q T_{*}}(a) i_{S T *}\left(b^{\prime}\right)-i_{Q T_{*}}(a) i_{R T *} i_{R Q}^{*}\left(a^{\prime}\right) \\
& +i_{S T *}(b) i_{Q T_{*}}\left(a^{\prime}\right)+i_{S T *}(b) i_{S T *}\left(b^{\prime}\right)-i_{S T *}(b) i_{R T *} i_{R Q}^{*}\left(a^{\prime}\right) \\
& -i_{R T *} i_{R Q}^{*}(a) i_{Q T_{*}}\left(a^{\prime}\right)-i_{R T *} i_{R Q}^{*}(a) i_{S T *}\left(b^{\prime}\right) \\
& +i_{R T *} i_{R Q}^{*}(a) i_{R T *} i_{R Q}^{*}\left(a^{\prime}\right) \\
& =\left(i_{Q T_{*}}(a)+i_{S T_{*}}(b)-i_{R T *} i_{R Q}^{*}(a)\right)\left(i_{Q T_{*}}\left(a^{\prime}\right)+i_{S T_{*}}\left(b^{\prime}\right)-i_{R T *} i_{R Q}^{*}\left(a^{\prime}\right)\right) \\
& =\beta(a, b) \beta\left(a^{\prime}, b^{\prime}\right) \text {. }
\end{aligned}
$$

This concludes the proof.

Proposition 4.6 can be generalized to the following situation. 
Theorem 4.7 Let $T$ be a toric complex, $T_{1}, \ldots, T_{r}$ be subcomplexes of $T$ such that $T=T_{1} \cup \cdots \cup T_{r}$ and let $\mathcal{P}(r)$ denote the partially ordered set consisting of all subsets of $\{1, \ldots, r\}$ ordered by inclusion. Given $I \in \mathcal{P}(r)$ we let $T_{I}$ denote the subcomplex $T_{I}=\cap_{i \in I} T_{i}$ of $T$. Then the natural homomorphism

$$
K[T] \rightarrow \lim _{I \in \mathcal{P}(r)} K\left[T_{I}\right]
$$

is an isomorphism.

Proof: We prove the lemma by induction on $r$, the case $r=1$ being obvious. Assume that the lemma holds for $r-1$. In particular, the homomorphisms

$$
K\left[T_{1} \cup \cdots \cup T_{r-1}\right] \rightarrow \lim _{I \in \mathcal{P}(r-1)} K\left[T_{I}\right] \text { and } K\left[\left(T_{1} \cup \cdots \cup T_{r-1}\right) \cap T_{r}\right] \rightarrow \lim _{I \in \mathcal{P}(r), r \in I} K\left[T_{I}\right]
$$

are isomorphisms. Since $T=\left(T_{1} \cup \cdots \cup T_{r-1}\right) \cup T_{r}$ there is a chain of isomorphisms

$$
\begin{aligned}
K[T] & \cong K\left[T_{1} \cup \cdots \cup T_{r-1}\right] \times \times_{K\left[\left(T_{1} \cup \cdots \cup T_{r-1}\right) \cap T_{r}\right]} K\left[T_{r}\right] \\
& \cong \lim _{I \in \mathcal{P}(r), r \notin I} K\left[T_{I}\right] \times \lim _{I \in \mathcal{P}(r), r \in I} K\left[T_{I}\right] K\left[T_{r}\right] \\
& \cong \lim _{I \in \mathcal{P}(r)} K\left[T_{I}\right] .
\end{aligned}
$$

where the first is the isomorphism of 4.6, the second isomorphism is induced by the isomorphisms that hold by the inductive hypothesis and the third isomorphism uses the fact that the objects in question have the same universal property.

For $F \in \Pi_{T}$ consider the complex $T(F)$ of Example 3.2. The face ring of $T(F)$ has the form $K[T(F)]=K[F] / \mathcal{I}_{F}$ where $\mathcal{I}_{F}$ is the kernel of the homomorphism $K[F] \rightarrow K\left[\mathbb{Z}^{d_{F}}\right]$. The following corollary was proved in [10, Prop. 4.8] by considering the limit as a subset of a product.

Corollary 4.8 Let $T$ be a toric complex. Then

$$
K[T] \cong \lim _{F \in \Pi_{T}^{\mathrm{op}}} K[T(F)]
$$

Proof: Note that $T(F) \cap T(G)=T(F \cap G)$ and apply 4.7 to the family $\{T(F)\}_{F \in \Pi_{T}}$.

Thus we have another representation of the face ring of a toric complex. For example this implies easily that $K[T]$ is reduced, because $K[T(F)]$ is reduced for $F \in \Pi_{T}$ and the limit of reduced rings is reduced. The next goal will be to interpret the Veronese subrings of the 
face ring of a standard graded toric complex. The following construction will be used later to subdivide simplicial toric complexes.

Definition 4.9 Given a toric complex $T$ and $r \geq 1$ we let $r T$ consist of the data:

(a) $\Pi_{r T}$ is the partially ordered set consisting of the sets

$$
r F=\left\{u \in \mathbb{N}^{F}: \sum_{a \in F} u(a)=r\right\} / \sim
$$

where $F \in \Pi_{T}$ and $u \sim v$ if $T_{F}(u)=T_{F}(v)$.

(b) For $r F \in \Pi_{r T}$ and $u \in r F$ let $r T_{r F}(u)=T_{F}(u)$.

(c) For $r F, r G \in \Pi_{r T}$ with $r F \subseteq r G$ let $r T_{r F, r G}=T_{F, G}$.

Proposition 4.10 Let $T$ be a toric complex and $r \geq 1$ a positive integer. Then $r T$ is a toric complex.

Proof: The sets $r F \in \Pi_{r T}$ are all subsets of the set $\{u: \operatorname{Gen}(T) \rightarrow \mathbb{N}\} / \sim$ where $u \sim v$ if there exists $G \in \Pi_{T}$ such that both $\operatorname{supp}(u)$ and $\operatorname{supp}(v)$ are contained in $G$ and $T_{G}(u)=T_{G}(v)$. By construction $r T_{r F}: r F \rightarrow \mathbb{Z}^{d_{F}} \backslash\{0\}$ is injective and $r T_{r F r G}=T_{F G}$ is an injection of abelian groups. We have to verify conditions (i)-(iv) of the definition of a toric complex. Condition (i) holds because we have that $r F \cap r G=r(F \cap G)$ for $F, G \in \Pi_{T}$. Conditions (ii) and (iii) are satisfied by construction, and condition (iv) holds, because cone $\left(T_{F}(F)\right)=\operatorname{cone}\left(r T_{r F}(r F)\right)$ for $F \in \Pi_{T}$.

Clearly if $T$ is a toric complex and $S$ is a subcomplex of $T$ then $r S$ is a subcomplex of $r T$.

Theorem 4.11 Let $T$ be a standard graded toric complex and $r \geq 1$ a positive number. Then $K[r T]=K[T]^{(r)}$ is the $r$-th Veronese ring of $K[T]$.

Proof: We compute

$$
K[r T] \cong \lim _{r F \in \Pi_{r T}^{\mathrm{op}}} K[(r T)(r F)] \cong \lim _{F \in \Pi_{T}^{\mathrm{op}}} K[T(F)]^{(r)} \cong K[T]^{(r)}
$$

where the second isomorphism follows from the definition of the toric complex $r T$.

\section{Subdivisions of toric complexes}

In this section we introduce and study subdivisions of toric complexes. In particular, we will relate the face rings of a toric complex and its regular subdivisions. 
Definition 5.1 Let $S$ and $T$ be a toric complexes.

(i) $S$ is a partial subdivision of $T$ if the following is satisfied:

(a) $\operatorname{Gen}(S) \subseteq \operatorname{Gen}(T)$,

(b) for all $F \in \Pi_{S}$ there exists a face of $T$ containing $F$,

(c) $S_{F}(a)=T_{F^{\prime}}(a)$ for all $F \in \Pi_{S}$ and $a \in F$ where $F^{\prime}=\bigcap_{F \subseteq G, G \in \Pi_{T}} G$,

(d) for $F, G \in \Pi_{S}$ with $F \subseteq G$ we have $S_{F, G}=T_{F^{\prime} G^{\prime}}$.

(ii) $S$ is a subdivision of $T$ if $S$ is a partial subdivision of $T$ satisfying that $\operatorname{cone}\left(T_{G}(G)\right)=$ $\bigcup_{F \in \Pi_{S}, F \subseteq G} \operatorname{cone}\left(T_{G}(F)\right)$ for $G \in \Pi_{T}$.

(iii) $S$ is a triangulation of $T$ if $S$ is simplicial and a subdivision of $T$.

In particular, if $S$ is a subdivision of $T$, then

$$
\|T\|=\underset{G \in \Pi_{T}}{\lim } \operatorname{cone}\left(T_{G}(G)\right)=\underset{G \in \Pi_{T}}{\lim _{F \in \Pi_{S}, F \subseteq G}} \bigcup_{\overrightarrow{F \in \Pi_{S}}} \operatorname{cone}\left(T_{G}(F)\right) \cong \underset{\lim }{\operatorname{lin}} \operatorname{cone}\left(S_{F}(F)\right)=\|S\|,
$$

and similarly we see that $|T| \cong|S|$.

Example 5.2 Given $F \subseteq \mathbb{Z}^{d} \backslash\{0\}$ and $a \in F$ we construct an embedded toric complex $T(F, a)$ such that $T(F, a)$ is a subdivision of the toric complex $T(F)$ of Example 3.2 with $\operatorname{Gen}(T(F, a))=\operatorname{Gen}(T(F))$. A subset $G$ of $F$ is in $\Pi_{T(F, a)}$ if there exists a face $H$ of $F$ in $T(F)$ such that $a \notin H$ and $G=\operatorname{cone}(H \cup\{a\}) \cap F$ or $G=H$. We leave it as an instructive exercise for the reader to check that $T(F, a)$ is a subdivison of $T(F)$.

Example 5.3 Let $T$ be an embedded toric complex and let $a \in \operatorname{Gen}(T)$. For a face $F$ of $T$ with $a \in F$ we have defined the subdivision $T(F, a)$ of $T(F)$. If $a \notin F$ we let $T(F, a)=T(F)$. The embedded toric complex $S$ with $\Pi_{S}=\cup_{F \in \Pi_{T}} \Pi_{T(F, a)}$ is a subdivision of $T$. In the case where $T=T\left(\Sigma,\left(G_{\sigma}\right)_{\sigma \in \Sigma}, \varphi\right)$ for a fan $\Sigma$ in $N$ as in Example 3.5, the subdivision $S$ of $T$ is well-known [12, p. 48].

The following construction is inspired by the way Sturmfels [23] subdivides the cone generated by a finite subset $F$ of $\mathbb{Z}^{d}$. We have modified Sturmfels' construction slightly so that the subdivision of a toric complex is again a toric complex.

Definition 5.4 Let $T$ be a toric complex and $\omega: \operatorname{Gen}(T) \rightarrow \mathbb{R}$. Then the $\omega$-subdivision $\operatorname{sd}_{\omega} T$ of $T$ is given by the following data:

(i) A subset $F$ of $\operatorname{Gen}(T)$ is in $\Pi_{\mathrm{sd}_{\omega} T}$ if there exists a face of $T$ containing $F$ and letting $F^{\prime}=\bigcap_{F \subseteq G \in \Pi_{T}} G$ there exists a linear form $\alpha_{F}$ on $\mathbb{R}^{d_{F^{\prime}}}$ such that $F=\{a \in$ $\left.F^{\prime}: \alpha_{F}\left(T_{F^{\prime}}(a)\right)=\omega(a)\right\}$ and $\alpha_{F}\left(T_{F^{\prime}}(a)\right) \leq \omega(a)$ for every $a \in F^{\prime}$, 
(ii) $\left(\operatorname{sd}_{\omega} T\right)_{F}(a)=T_{F^{\prime}}(a)$ for $F \in \Pi_{\mathrm{sd}_{\omega} T}$ and $F^{\prime}=\bigcap_{F \subseteq G \in \Pi_{T}} G$,

(iii) $\left(\operatorname{sd}_{\omega} T\right)_{F G}=T_{F^{\prime} G^{\prime}}$.

If $\operatorname{sd}_{\omega} T$ is a subdivision (triangulation) of $T$, we say that $\operatorname{sd}_{\omega} T$ is a regular subdivision (triangulation) of $T$.

A standard example of a non-regular triangulation is given in [23, Example 8.2]. Let $e_{1}, \ldots, e_{d+1}$ be the standard basis for $\mathbb{R}^{d+1}$. A face $F$ of a cone $C \subset \mathbb{R}^{d+1}$ is called a lower face if for all $x \in F$ and $r>0$ we have that $x-r \cdot e_{d+1} \notin F$. It is easy to see that $F$ is a lower face if and only if it is the intersection of $C$ with a hyperplane $H_{\alpha}(0)$ such that $\alpha\left(e_{d+1}\right)<0$ and $C \subseteq H_{\alpha}^{-}(0)$.

Remark 5.5 Observe that (i) of Definition 5.4 can be reformulated as follows: A subset $F$ of $\operatorname{Gen}(T)$ is in $\Pi_{\mathrm{sd}_{\omega} T}$ if cone( $\left.\operatorname{sd}_{\omega} T_{F^{\prime}}(F)\right)$ is the projection of a lower face of cone $\left(\left(T_{F^{\prime}}(b), w(b)\right): b \in F^{\prime}\right) \subset \mathbb{R}^{d_{F^{\prime}+1}}$ with respect to the last coordinate.

Proposition 5.6 $\operatorname{sd}_{\omega} T$ is a partial subdivision of $T$ for every toric complex $T$ and every $\omega: \operatorname{Gen}(T) \rightarrow \mathbb{R}$.

Proof: (a), (b) and (c) of the definition of a toric complex are clearly satisfied. It remains to verify conditions (i)-(iv). (ii) and (iii) are fulfilled by definition.

(i): Let $F, G \in \Pi_{\text {sd }_{\omega} T}$. Choose $F^{\prime}=\cap_{F \subseteq F^{\prime \prime} \in \Pi_{T}} F^{\prime \prime}, \alpha_{F}: \mathbb{R}^{d_{F^{\prime}}} \rightarrow \mathbb{R}$ and $G^{\prime}=\cap_{G \subseteq G^{\prime \prime} \in \Pi_{T}}$ $G^{\prime \prime}, \alpha_{G}: \mathbb{R}^{d_{G^{\prime}}} \rightarrow \mathbb{R}$ as in Definition 5.4. $F^{\prime} \cap G^{\prime}$ is an element of $\Pi_{T}$. Via the inclusion of $\mathbb{R}^{d_{F^{\prime} \cap G^{\prime}}}$ in $\mathbb{R}^{d_{F^{\prime}}}$ induced by $T_{F^{\prime} \cap G^{\prime} F^{\prime}}$ the linear form $\alpha_{F}$ on $\mathbb{R}^{d_{F^{\prime}}}$ induces a linear form $\beta_{F}$ on

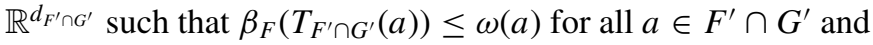

$$
F \cap G^{\prime}=\left\{a \in F^{\prime} \cap G^{\prime}: \beta_{F}\left(T_{F^{\prime} \cap G^{\prime}}(a)\right)=\omega(a)\right\} .
$$

Analogously there exists a linear form $\beta_{G}$ on $\mathbb{R}^{d_{F^{\prime} \cap G^{\prime}}}$ such that $\beta_{G}\left(T_{F^{\prime} \cap G^{\prime}}(a)\right) \leq \omega(a)$ for all $a \in F^{\prime} \cap G^{\prime}$ and

$$
F^{\prime} \cap G=\left\{a \in F^{\prime} \cap G^{\prime}: \beta_{G}\left(T_{F^{\prime} \cap G^{\prime}}(a)\right)=\omega(a)\right\} .
$$

Let $\alpha_{F \cap G}:=\left(\beta_{F}+\beta_{G}\right) / 2: \mathbb{R}^{d_{F^{\prime} \cap G^{\prime}}} \rightarrow \mathbb{R}$. Then

$$
\alpha_{F \cap G}\left(T_{F^{\prime} \cap G^{\prime}}(a)\right) \leq \omega(a) \text { for all } a \in F^{\prime} \cap G^{\prime}
$$

and

$$
F \cap G=\left\{a \in F^{\prime} \cap G^{\prime}: \alpha_{F \cap G}\left(T_{F^{\prime} \cap G^{\prime}}(a)\right)=\omega(a)\right\} .
$$

Hence $F \cap G \in \Pi_{\text {sd }_{\omega} T}$ and this shows (i).

(iv): Assume that $F \subseteq G \in \Pi_{\mathrm{sd}_{\omega} T}$. If $F \in \Pi_{\mathrm{sd}_{\omega} T}$, then choose $F^{\prime}, \alpha_{F}: \mathbb{R}^{d_{F^{\prime}}} \rightarrow \mathbb{R}$ and $G^{\prime}$, $\alpha_{G}: \mathbb{R}^{d_{G^{\prime}}} \rightarrow \mathbb{R}$ as in the proof of (i). Note that $F^{\prime} \subseteq G^{\prime}$. Therefore cone $\left(T_{G^{\prime}}\left(F^{\prime}\right)\right)=H_{\gamma}(0) \cap$ 
cone $\left(T_{G^{\prime}}\left(G^{\prime}\right)\right)$ is a face of cone $\left(T_{G^{\prime}}\left(G^{\prime}\right)\right)$ for some linear form $\gamma$ on $\mathbb{R}^{d_{G^{\prime}}}$, cone $\left(T_{G^{\prime}}\left(G^{\prime}\right)\right) \subseteq$ $H_{\gamma}^{-}(0)$ and $T_{G^{\prime}}\left(F^{\prime}\right)=\operatorname{cone}\left(T_{G^{\prime}}\left(F^{\prime}\right)\right) \cap T_{G^{\prime}}\left(G^{\prime}\right)$. We have the inclusion $\mathbb{R}^{d_{F^{\prime}}} \rightarrow \mathbb{R}^{d_{G^{\prime}}}$ and we extend $\alpha_{F}$ arbitrarily to a linear form on $\mathbb{R}^{d_{G^{\prime}}}$.

Now choose $t \gg 0$ such that for $\beta_{F}=\alpha_{F}+t \cdot \gamma$ we have that $F=\left\{a \in G^{\prime}: \beta_{F}\left(T_{G^{\prime}}(a)\right)=\right.$ $\omega(a)\}$ and $\beta_{F}\left(T_{G^{\prime}}(a)\right) \leq \omega(a)$ for every $a \in G^{\prime}$. Thus $\beta=\beta_{F}-\alpha_{G}$ is a linear form such that cone $\left(T_{G^{\prime}}(F)\right)=H_{\beta}(0) \cap \operatorname{cone}\left(T_{G^{\prime}}(G)\right)$ is a face of cone $\left(T_{G^{\prime}}(G)\right)$ and $T_{G^{\prime}}(F)=$ $\operatorname{cone}\left(T_{G^{\prime}}(F)\right) \cap T_{G^{\prime}}(G)$.

Conversely, assume that $F \subseteq G \in \Pi_{\mathrm{sd}_{\omega} T}, G^{\prime}, \alpha_{G}: \mathbb{R}^{d_{G^{\prime}}} \rightarrow \mathbb{R}$ are chosen as above and $\operatorname{cone}\left(T_{G^{\prime}}(F)\right)=H_{\beta}(0) \cap \operatorname{cone}\left(T_{G^{\prime}}(G)\right)$ is a face of cone $\left(T_{G^{\prime}}(G)\right)$ defined by some linear form $\beta$ on $\mathbb{R}^{d_{G^{\prime}}}$ and $T_{G^{\prime}}(F)=\operatorname{cone}\left(T_{G^{\prime}}(F)\right) \cap T_{G^{\prime}}(G)$. Define $\beta_{F}=\alpha_{G}+t \cdot \beta$. For a suitable $t \geq 0$ we have that $F=\left\{a \in G^{\prime}: \beta_{F}\left(T_{G^{\prime}}(a)\right)=\omega(a)\right\}$ and $\beta_{F}\left(T_{G^{\prime}}(a)\right) \leq \omega(a)$ for every $a \in G^{\prime}$. This implies that $F \in \Pi_{\operatorname{sd}_{\omega} T}$ and we are also done in this case.

Hence $\operatorname{sd}_{\omega} T$ is a toric complex which is a partial subdivision of $T$ by construction.

If $S$ is a subcomplex of a toric complex $T$, then a map $\omega: \operatorname{Gen}(T) \rightarrow \mathbb{R}$ induces a map $\omega: \operatorname{Gen}(S) \rightarrow \mathbb{R}$.

Corollary 5.7 Let $T$ be a toric complex, $\omega: \operatorname{Gen}(T) \rightarrow \mathbb{R}$ and $S$ a subcomplex of $T$. Then $\operatorname{sd}_{\omega} S$ is a subcomplex of $\operatorname{sd}_{\omega} T$ and if $\operatorname{sd}_{\omega} T$ is a regular subdivision (triangulation) of $T$, then $\operatorname{sd}_{\omega} S$ is a regular subdivision (triangulation) of $S$.

Proof: This follows from Definition 5.4 since the property to be a regular subdivision is defined on the faces of a toric complex.

A subcomplex of a toric complex inherits regular subdivisions as noted in 5.7. The corresponding face rings are related as follows.

Corollary 5.8 Let $T$ be a toric complex, $\omega: \operatorname{Gen}(T) \rightarrow \mathbb{R}$ such that $\operatorname{sd}_{\omega} T$ is a regular subdivision of $T$ and $S$ a subcomplex of $T$. Then

$$
K\left[\operatorname{sd}_{\omega} S\right] \cong K\left[\operatorname{sd}_{\omega} T\right] /\left(x_{F}: F \subseteq \operatorname{Gen}\left(\operatorname{sd}_{\omega} T\right) \text { is not contained in any } G \in \Pi_{\operatorname{sd}_{\omega} S}\right)
$$

Proof: By Corollary 5.7 the toric complex $\operatorname{sd}_{\omega} S$ is a regular subdivision of $S$ and a subcomplex of $\operatorname{sd}_{\omega} T$. The isomorphisms follow from Proposition 4.2.

The toric complex $\operatorname{sd}_{\omega} T$ is not always a subdivision of $T$. As an illustration let $F$ denote the finite set $F=\{-1,1\} \subseteq \mathbb{Z}$, let $\omega: F \rightarrow \mathbb{R}$ be the constant function with value -1 and consider the toric complex $T(F)$ of Example 3.2. Then $\|T(F)\|=\mathbb{R}$ and $\left\|\operatorname{sd}_{\omega} T(F)\right\|=\emptyset$, because $\Pi_{\mathrm{sd}_{\omega} T(F)}$ is the empty set.

Proposition 5.9 Let $T$ be a toric complex and $\omega: \operatorname{Gen}(T) \rightarrow \mathbb{R}$. Then $\operatorname{sd}_{\omega} T$ is a regular subdivision of $T$ if one of the following conditions is satisfied: 
(i) All values $\omega($ a) of $\omega$ are positive.

(ii) $T$ is an $\mathbb{N}$-graded toric complex.

Proof: In both cases it remains to show that

$$
\operatorname{cone}\left(T_{G}(G)\right)=\bigcup_{F \in \Pi_{\mathrm{sd}_{\omega} T}, F \subseteq G} \operatorname{cone}\left(T_{G}(F)\right)
$$

for $G \in \Pi_{T}$, because by 5.6 we know already that $\operatorname{sd}_{\omega} T$ is a partial subdivision of $T$. Let $G \in \Pi_{T}$ and $0 \neq x \in \operatorname{cone}\left(T_{G}(G)\right)$. Consider the cone

$$
C=\operatorname{cone}\left(\left(T_{G}(b), \omega(b)\right): b \in G\right) \subset \mathbb{R}^{d_{G}+1} \quad \text { and } \quad P=\{(x, t): t \in \mathbb{R}\} \subset \mathbb{R}^{d_{G}+1} .
$$

Since $x \in \operatorname{cone}\left(T_{G}(G)\right)$ we have that $C \cap P \neq \emptyset$. If one of the conditions (i) or (ii) is satisfied there exists

$$
s=\inf \{t \in \mathbb{R}:(x, t) \in P \cap C\}>-\infty .
$$

Then $(x, s)$ is an element of a lower face $F$ of $C$ and by Remark 5.5 there exists a linear form $\alpha$ on $\mathbb{R}^{d_{G}}$ and $F^{\prime} \in \Pi_{T}$ such that $\alpha_{F}\left(T_{F^{\prime}}(a)\right) \leq \omega(a)$ for every $a \in F^{\prime}$ with $x \in \operatorname{cone}(F)$ and $\left.F=\left\{a \in F^{\prime}: \alpha_{F}\left(T_{F^{\prime}}(a)\right)=\omega(a)\right)\right\}$.

Example 5.10 Let $T$ be a toric complex and consider the function $\omega$ : $\operatorname{Gen}(2 T) \rightarrow \mathbb{R}$ with $\omega\left(2 \chi_{a}\right)=2$ for $a \in \operatorname{Gen}(T)$ and $\omega(u)=1$ if $u$ is not of the form $2 \chi_{a}$. Here $\chi_{a}$ is the indicator function on $a$ with $\chi_{a}(a)=1$ and $\chi_{a}(b)=0$ for $b \in \operatorname{Gen}(T) \backslash\{a\}$. In the case, where $T=T\left(\Sigma,\left(G_{\sigma}\right)_{\sigma \in \Sigma}, \varphi\right)$ for a rational fan $\Sigma$, the toric complex $2 T$ is of the form $2 T=T\left(\Sigma,\left(H_{\sigma}\right)_{\sigma \in \Sigma}, \varphi\right)$, where $H_{\sigma}=\left\{a+b: a, b \in G_{\sigma}\right\}$ and the fan $\left\{\operatorname{cone}\left(T_{F}(F)\right): F \in \Pi_{s d_{\omega}(2 T)}\right\}$ is a subdivision of the fan $\Sigma$.

Next we construct a homomorphism

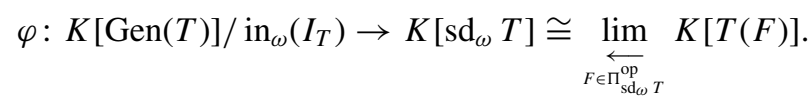

If we show that for all $F \in \Pi_{\operatorname{sd}_{\omega} T}$ the projection $i^{*}: K[\operatorname{Gen}(T)] \rightarrow K[F]$ induced by the inclusion $F \subseteq \operatorname{Gen}(T)$ induces a homomorphism $\varphi_{F}: K[\operatorname{Gen}(T)] / \operatorname{in}_{\omega}\left(I_{T}\right) \rightarrow K[T(F)]$, then $\varphi$ exists by the universal property of the limit.

Given $F \in \Pi_{\operatorname{sd}_{\omega} T}$ we let $F^{\prime}=\bigcap_{F \subseteq G \in \Pi_{T}} G \in \Pi_{T}$ as above. By the construction of $\operatorname{sd}_{\omega} T$, there exists a linear form $\alpha_{F}$ on $\mathbb{R}^{d_{F^{\prime}}}$ such that $F=\left\{a \in F^{\prime}: \alpha_{F}\left(T_{F^{\prime}}(a)\right)=\omega(a)\right\}$ and $\alpha_{F}\left(T_{F^{\prime}}(a)\right) \leq \omega(a)$ for $a \in F^{\prime}$. Note that $\omega$ induces a weight order on $K\left[F^{\prime}\right]$. For $x^{u} \in K\left[F^{\prime}\right]$ we have that

$$
\omega(u)=\sum_{a \in F^{\prime}} u(a) \omega(a) \geq \sum_{a \in F^{\prime}} u(a) \alpha_{F}\left(T_{F^{\prime}}(a)\right)=\alpha_{F}\left(T_{F^{\prime}}(u)\right)
$$


and equality holds precisely if $\operatorname{supp}(u) \subseteq F$. Let $x^{v} \in K\left[F^{\prime}\right]$ with $T_{F^{\prime}}(u)=T_{F^{\prime}}(v)$. If

$$
\sum_{a \in F^{\prime}} u(a) \omega(a)=\sum_{a \in F^{\prime}} v(a) \omega(a),
$$

then $\operatorname{supp}(u) \subseteq F$ if and only if $\operatorname{supp}(v) \subseteq F$.

Let $g \in \mathcal{I}_{F^{\prime}} \subset K\left[F^{\prime}\right]$. We show that the projection of $\operatorname{in}_{\omega}(g)$ to $K[F]$ is an element of

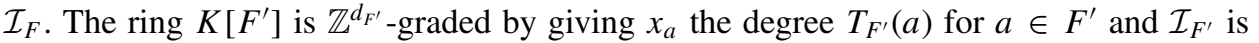
homogeneous with respect to this grading. Thus without loss of generality we may assume that there exists $z \in \mathbb{Z}^{d_{F^{\prime}}}$ such that $g=\sum_{u} c_{u} x^{u}$ and $T_{F^{\prime}}(u)=z$ for $c_{u} \neq 0$.

By the above discussion either in $\operatorname{in}_{\omega}(g)=g$ or for all monomials $x^{u}$ in in $_{\omega}(g)$ we have $\operatorname{supp}(u) \nsubseteq F$. In the first case the image of $\operatorname{in}_{\omega}(g)=g$ under the projection $i^{*}: K\left[F^{\prime}\right] \rightarrow$ $K[F]$ is in $\mathcal{I}_{F}$. In the second case $i^{*}\left(\operatorname{in}_{\omega}(g)\right)=0$. We obtain that $i^{*} K\left[F^{\prime}\right] \rightarrow K[F]$ induces a homomorphism $\varphi_{F F^{\prime}}: K\left[F^{\prime}\right] / \operatorname{in}_{\omega}\left(\mathcal{I}_{F^{\prime}}\right) \rightarrow K[F] / \mathcal{I}_{F}$. The following diagram of natural projections commutes for $F \in \Pi_{\text {sd }_{\omega} T}$ :

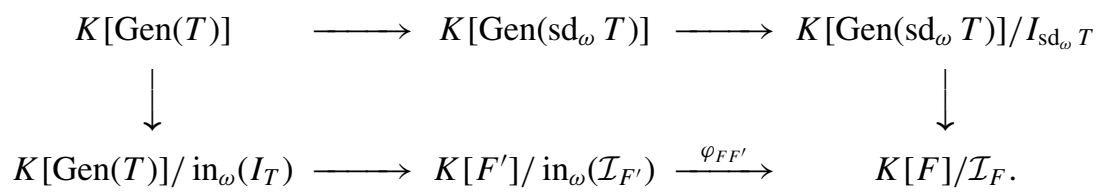

Taking limit with respect to $F$ we get a commutative diagram of the form:

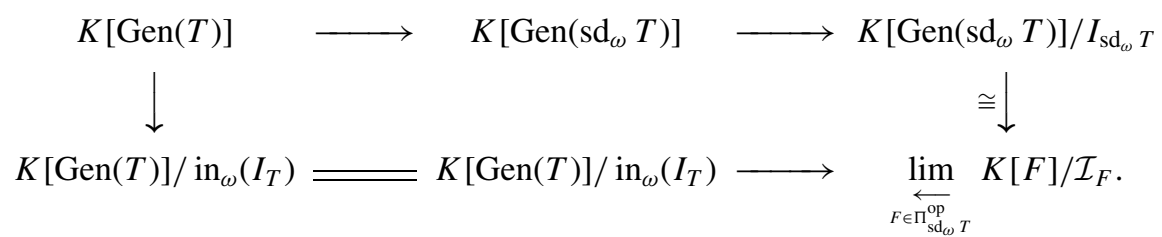

Thus the projection $K[\operatorname{Gen}(T)] \rightarrow K\left[\operatorname{Gen}\left(\operatorname{sd}_{\omega} T\right)\right]$ induces a homomorphism $\varphi$ from $K[\operatorname{Gen}(T)] / \operatorname{in}_{\omega}\left(I_{T}\right)$ to $K\left[\operatorname{sd}_{\omega} T\right]$. Since $K\left[\operatorname{sd}_{\omega} T\right]$ is reduced we obtain a homomorphism $\Phi: K[\operatorname{Gen}(T)] / \operatorname{rad}\left(\operatorname{in}_{\omega}\left(I_{T}\right)\right) \rightarrow K\left[\operatorname{sd}_{\omega} T\right]$.

Theorem 5.11 For every toric complex $T$ and $\omega: \operatorname{Gen}(T) \rightarrow \mathbb{R}$ such that $\operatorname{sd}_{\omega} T$ is a regular subdivision of $T$ the homomorphism $\Phi: K[\operatorname{Gen}(T)] / \operatorname{rad}\left(\operatorname{in}_{\omega}\left(I_{T}\right)\right) \rightarrow K\left[\operatorname{sd}_{\omega} T\right]$ is an isomorphism.

Proof: Recall that $I_{\mathrm{sd}_{\omega} T}=J_{\mathrm{sd}_{\omega} T}+\sum_{F \in \Pi_{\mathrm{sd}_{\omega} T}} I_{F}$ and $I_{T}=J_{T}+\sum_{G \in \Pi_{T}} I_{G}$.

We start by showing that the inclusion $i_{*}: K\left[\operatorname{Gen}\left(\operatorname{sd}_{\omega} T\right)\right] \rightarrow K[\operatorname{Gen}(T)]$ maps $I_{\mathrm{sd}_{\omega} T}$ into $\operatorname{in}_{\omega}\left(I_{T}\right)$. We identify monomials in $K\left[\operatorname{Gen}\left(\operatorname{sd}_{\omega} T\right)\right]$ with the corresponding ones in $K[\operatorname{Gen}(T)]$. For every $F \in \Pi_{\mathrm{sd}_{\omega} T}$ there exists $F^{\prime} \in \Pi_{T}$ with $F \subseteq F^{\prime}$, and a linear from $\alpha_{F}$ on $\mathbb{R}^{d_{F^{\prime}}}$ with $\alpha_{F}\left(T_{F^{\prime}}(a)\right)=\omega(a)$ for $a \in F$ and $\alpha_{F}\left(T_{F^{\prime}}(a)\right)<\omega(a)$ for $a \in F^{\prime} \backslash F$. The (image of a) binomial $x^{u}-x^{v} \in \mathcal{I}_{F}$ is an element of $I_{F^{\prime}}$. (Recall that these binomials 
generate $I_{F}$.) Since $\operatorname{supp}(u), \operatorname{supp}(v) \subseteq F$ we have

$$
\begin{aligned}
\omega(u) & =\sum_{a \in F} u(a) \omega(a)=\sum_{a \in F} u(a) \alpha_{F}\left(T_{F^{\prime}}(a)\right)=\alpha_{F}\left(T_{F^{\prime}}(u)\right)=\alpha_{F}\left(T_{F^{\prime}}(v)\right) \\
& =\sum_{a \in F} v(a) \alpha_{F}\left(T_{F^{\prime}}(a)\right)=\sum_{a \in F} v(a) \omega(a)=\omega(v),
\end{aligned}
$$

thus $x^{u}-x^{v}$ is an element of $\operatorname{in}_{\omega}\left(I_{F^{\prime}}\right) \subseteq \operatorname{rad}\left(\operatorname{in}_{\omega}\left(I_{T}\right)\right)$.

If $x^{u} \in J_{\operatorname{sd}_{\omega} T}$, then either $x^{u}$ is in $J_{T}$ and we are done, or there exists an $G \in \Pi_{T}$ such that $\operatorname{supp}\left(x^{u}\right) \subseteq G$. Suppose that $x^{u} \in J_{\text {sd }_{\omega} T}$ and that $\operatorname{supp}\left(x^{u}\right) \subseteq G \in \Pi_{T}$. Since $\operatorname{sd}_{\omega} T$ is a subdivision of $T$ and $T_{G}(u) \in \operatorname{cone}\left(T_{G}(G)\right)$ there exists $F \in \Pi_{\text {sd }_{\omega} T}$ with $F \subseteq G$ such that $T_{G}(u) \in \operatorname{cone}\left(T_{G}(F)\right)$. We may assume that $G=F^{\prime}$ and then there exists a linear from $\alpha_{F}$ on $\mathbb{R}^{d_{F^{\prime}}}$ with $\alpha_{F}\left(T_{F^{\prime}}(a)\right)=\omega(a)$ for $a \in F$ and $\alpha_{F}\left(T_{F^{\prime}}(a)\right)<\omega(a)$ for $a \in F^{\prime} \backslash F$.

Since $T_{F^{\prime}}(u) \in \operatorname{cone}\left(T_{F^{\prime}}(F)\right)$ there exists a map $\lambda: F \rightarrow \mathbb{R}_{+}$such that $T_{F^{\prime}}(u)=$ $\sum_{a \in F} \lambda(a) T_{F^{\prime}}(a)$. Using for example that Farkas lemma holds over both $\mathbb{R}$ and $\mathbb{Q}$, we may assume that $\lambda$ takes values in $\mathbb{Q}_{+}$, and thus there exists $v: F \rightarrow \mathbb{N}$ and $n \in \mathbb{N}$ such that $T_{F^{\prime}}(n u)=T_{F^{\prime}}(v)$. Now consider $x^{n u}-x^{v} \in I_{F^{\prime}}$. There exists at least one $a \in \operatorname{supp}(u)$ with $\alpha_{F}\left(T_{F^{\prime}}(a)\right)<\omega(a)$ since $x^{u} \in J_{\operatorname{sd}_{\omega} T}$. Since $\operatorname{supp}(v) \subseteq F$ we have

$$
\omega(n u)>\alpha_{F}\left(T_{F^{\prime}}(n u)\right)=\alpha_{F}\left(T_{F^{\prime}}(v)\right)=\omega(v) .
$$

We conclude that $x^{n u} \in \operatorname{in}_{\omega}\left(I_{F^{\prime}}\right) \subseteq \operatorname{in}_{\omega}\left(I_{T}\right)$. Hence $x^{u} \in \operatorname{rad}\left(\operatorname{in}_{\omega}\left(I_{T}\right)\right)$. This finishes the proof of the fact that the inclusion $i_{*}: K\left[\operatorname{Gen}\left(\operatorname{sd}_{\omega} T\right)\right] \rightarrow K[\operatorname{Gen}(T)] \operatorname{maps} I_{\mathrm{sd}_{\omega} T}$ into $\operatorname{rad}\left(\operatorname{in}_{\omega}\left(I_{T}\right)\right)$.

We denote by $\Psi: K\left[\operatorname{Gen}\left(\operatorname{sd}_{\omega} T\right)\right] / I_{\mathrm{sd}_{\omega} T} \rightarrow K[\operatorname{Gen}(T)] / \operatorname{rad}\left(\operatorname{in}_{\omega}\left(I_{T}\right)\right)$ the map induced by $i_{*}$. It is immediate that $\Phi \circ \Psi=\operatorname{id}_{K\left[\operatorname{sd}_{\omega} T\right]}$. We need to prove that $\Psi \circ \Phi$ is the identity, or equivalently that $\Psi$ is onto. Since $\operatorname{sd}_{\omega} T$ is a subdivision of $T$ there exists for every $G \in \Pi_{T}$ and $a \in G$ an $F \subseteq G$ with $F \in \Pi_{\text {sd }_{\omega} T}$ such that $T_{G}(a) \in \operatorname{cone}\left(T_{G}(F)\right)$. Again we may assume that $G=F^{\prime}$ and $\alpha_{F^{\prime}}: \mathbb{R}^{d_{F^{\prime}}} \rightarrow \mathbb{R}$ is chosen as above. If $x_{a}$ is not in the image of the inclusion $i_{*}: K\left[\operatorname{Gen}\left(\operatorname{sd}_{\omega} T\right)\right] \rightarrow K[\operatorname{Gen}(T)]$, then $a$ is not in $F$ and $\alpha_{F}\left(T_{F^{\prime}}(a)\right)<\omega(a)$. Writing $n \cdot T_{F^{\prime}}(a)=\sum_{b \in F} v(b) T_{F^{\prime}}(b)$ for $n \in \mathbb{N}$ and $v: F \rightarrow \mathbb{N}$ as above, we compute that $x_{a}^{n}-x^{v} \in I_{F^{\prime}}$, and $\omega(n a)>\alpha_{F}\left(n \cdot T_{F^{\prime}}(a)\right)=\alpha_{F}\left(T_{F^{\prime}}(v)\right)=\omega(v)$. Hence $x_{a}^{n} \in \operatorname{in}_{\omega}\left(I_{F^{\prime}}\right)$. It follows that $x_{a} \in \operatorname{rad}\left(\operatorname{in}_{\omega}\left(I_{F^{\prime}}\right)\right) \subseteq \operatorname{rad}\left(\operatorname{in}_{\omega}\left(I_{T}\right)\right)$ for every $a \in \operatorname{Gen}(T) \backslash \operatorname{Gen}\left(\operatorname{sd}_{\omega} T\right)$. This finishes the proof of the theorem.

Theorem 5.11 generalizes a result of Sturmfels [23, Theorem 8.3]:

Corollary 5.12 Let $T$ be a toric complex and $\omega: \operatorname{Gen}(T) \rightarrow \mathbb{R}$ such that $\operatorname{sd}_{\omega} T$ is a regular subdivision of $T$. Then

(i) $\operatorname{rad}\left(\operatorname{in}_{\omega}\left(I_{T}\right)\right)$ is a square-free monomial ideal if and only if $\operatorname{sd}_{\omega} T$ is a regular triangulation of $T$. In this case the abstract simplicial complex induced by $\operatorname{rad}\left(\operatorname{in}_{\omega}\left(I_{T}\right)\right)$ coincides with $\Pi_{\mathrm{sd}_{\omega} T}$.

(ii) Given $\omega^{\prime}: \operatorname{Gen}(T) \rightarrow \mathbb{R}$ such that $\operatorname{sd}_{\omega^{\prime}} T$ is a subdivision of $T$ we have that $\operatorname{sd}_{\omega} T=$ $\operatorname{sd}_{\omega^{\prime}} T$ if and only if $\operatorname{rad}\left(\operatorname{in}_{\omega}\left(I_{T}\right)\right)=\operatorname{rad}\left(\operatorname{in}_{\omega^{\prime}}\left(I_{T}\right)\right)$. 
Proof: (i): By Theorem 5.11 $I_{\mathrm{sd}_{\omega} T}$ is a monomial ideal if and only if $\operatorname{rad}\left(\operatorname{in}_{\omega}\left(I_{T}\right)\right)$ is a square-free monomial ideal. Hence it follows from 4.5 that $\operatorname{rad}\left(\operatorname{in}_{\omega}\left(I_{T}\right)\right)$ is a square-free monomial ideal if and only if $\operatorname{sd}_{\omega} T$ is a regular triangulation of $T$ and that the abstract simplicial complex induced by $\operatorname{rad}\left(\operatorname{in}_{\omega}\left(I_{T}\right)\right)$ coincides with $\Pi_{\mathrm{sd}_{\omega} T}$.

(ii): We only show that $\operatorname{rad}\left(\operatorname{in}_{\omega}\left(I_{T}\right)\right)=\operatorname{rad}\left(\operatorname{in}_{\omega^{\prime}}\left(I_{T}\right)\right)$ implies that $\operatorname{sd}_{\omega} T=\operatorname{sd}_{\omega^{\prime}} T$ since the other implication is a direct consequence of 5.11. Observe that by 5.11 we have that $\operatorname{rad}\left(\operatorname{in}_{\omega}\left(I_{T}\right)\right)=\operatorname{rad}\left(\operatorname{in}_{\omega^{\prime}}\left(I_{T}\right)\right)$ if and only if $\operatorname{Gen}\left(\operatorname{sd}_{\omega} T\right)=\operatorname{Gen}\left(\operatorname{sd}_{\omega^{\prime}} T\right)$ and $I_{\mathrm{sd}_{\omega} T}=I_{\mathrm{sd}_{\omega^{\prime}} T}$. The result follows from the fact that for a monomial $x^{u}$ the support of $u$ is contained in a face of $\operatorname{sd}_{\omega} T$ if and only if $x^{u} \notin I_{\mathrm{Sd}_{\omega} T}$.

Recall that given a subset $L$ of $\mathbb{Z}^{d}$ we let $M_{L}$ denote the submonoid of $\mathbb{Z}^{d}$ generated by $L$. Let $L \subseteq L^{\prime}$ be subsets of $\mathbb{Z}^{d}$. We say that $M_{L}$ is integrally closed in $M_{L^{\prime}}$ if for all $x \in M_{L^{\prime}}$ with $n \cdot x \in M_{L}$ for some $n \in \mathbb{N}$ we have that $x \in M_{L}$. It is easy to see that this is equivalent to the fact that $\operatorname{cone}(L) \cap M_{L^{\prime}}=M_{L}$.

The following proposition can be extracted from the proof of Theorem 5.11:

Proposition 5.13 Let $T$ be a toric complex and $\omega: \operatorname{Gen}(T) \rightarrow \mathbb{R}$. If $\operatorname{Gen}\left(\operatorname{sd}_{\omega} T\right)=$ $\operatorname{Gen}(T)$ and $T_{G}(F)$ is integrally closed in $T_{G}(G)$ for all $F \in \Pi_{\operatorname{sd}_{\omega} T}$ and $G \in \Pi_{T}$ with $F \subseteq G$, then the ideal $\operatorname{in}_{\omega}\left(I_{T}\right)$ is a radical ideal.

\section{Edgewise subdivision of a simplicial complex}

In this section $\Delta$ denotes an abstract simplicial complex on the vertex set $V=\{1, \ldots, d+1\}$, i.e. $\Delta$ is a set of subsets of $V$ and $F \subseteq G$ for $G \in \Delta$ implies that $F \in \Delta$. Let $K[\Delta]=$ $K[V] / I_{\Delta}$ be the Stanley-Reisner ring of $\Delta$ where $I_{\Delta}=\left(x_{F}: F \subset\{1, \ldots, d+1\}, F \notin \Delta\right)$ is the Stanley-Reisner ideal of $\Delta$. Observe that $K[\Delta]=K[T(\Delta)]$ where $T(\Delta)$ is the toric complex of 3.3. By Theorem 4.11 the ring $K[r T(\Delta)]=K[T(\Delta)]^{(r)}$ is the $r$-th Veronese subring of $K[T(\Delta)]$. Given an element $m$ of $\mathbb{Z}^{d+1}$ we let $m_{j}$ denote its $j$-th coordinate for $1 \leq j \leq d+1$.

Guided by the regular subdivision introduced by Knudsen and Mumford [15, pp. 117123 ] and the edgewise subdivision of simplicial sets (see for example [2, 8] or [13]) we define the following simplicial complex.

Definition 6.1 Let $\Delta$ be an abstract simplicial complex with vertex set $V$, let $r \geq 1$ and $\iota_{V}: V \rightarrow \mathbb{Z}^{d+1}$ as in Example 3.3. Let $\iota_{V}$ also denote the map $\mathbb{N}^{V} \rightarrow \mathbb{Z}^{d+1}, u \mapsto$ $\sum_{i=1}^{d+1} u(i) \iota_{V}(i)$. The $r$-fold edgewise subdivision of $\Delta$ is the abstract simplicial complex $\operatorname{esd}_{r}(\Delta)$ consisting of the subsets $F \subseteq r V=\left\{u \in \mathbb{N}^{V}: \sum_{i \in V} u(i)=r\right\}$ with $\bigcup_{u \in F} \operatorname{supp}(u) \in \Delta$ satisfying that for $u, u^{\prime} \in F$ we either have that $0 \leq\left(\iota_{V}(u)-\iota_{V}\left(u^{\prime}\right)\right)_{j} \leq 1$ for every $j \in\{1, \ldots, d+1\}$ or that $0 \leq\left(\iota_{V}\left(u^{\prime}\right)-\iota_{V}(u)\right)_{j} \leq 1$ for every $j \in\{1, \ldots, d+1\}$.

The name $r$-fold edgewise subdivision comes from the fact that the edges of $\Delta$ are subdivided in $r$ pieces. The goal of this section will be to relate $K\left[\operatorname{esd}_{r}(\Delta)\right]$ to the $r$-th Veronese subring of the Stanley-Reisner ring $K[\Delta]$. 
Observe that we have required that the coordinate-wise partial order on $\mathbb{Z}^{d+1}$ induces a total order on $\iota_{V}(F)$ for every $F \in \operatorname{esd}_{r}(\Delta)$. If $m(F)$ denotes the minimal element of $\iota_{V}(F)$ in this order, then we also require that the $\operatorname{set} l_{V}(F) \subseteq \mathbb{Z}^{d+1}$ is a subset of $m(F)+\left(\{0,1\}^{d} \times\{0\}\right)$, the vertices of a $d$-dimensional lattice cube.

Given an element $\sigma$ of the symmetric group $\Sigma_{d}$ let

$$
\Delta_{\sigma}=\operatorname{conv}\left(0, e_{\sigma(1)}, e_{\sigma(1)}+e_{\sigma(2)}, \ldots, e_{\sigma(1)}+e_{\sigma(2)}+\cdots+e_{\sigma(d)}\right) \subseteq \mathbb{R}^{d+1},
$$

and denote by $\Delta^{d}$ the abstract simplex on the vertex set $V$. For every maximal face $F$ of $\operatorname{esd}_{r}\left(\Delta^{d}\right)$ the polytope $\operatorname{conv}\left(\iota_{V}(F)\right)$ is a simplex of the form $m(F)+\Delta_{\sigma(F)}$ for a unique $\sigma(F) \in \Sigma_{d}$. Conversely, for every $m \in \mathbb{Z}^{d+1}$ and $\sigma \in \Sigma_{d}$ such that $m+\Delta_{\sigma}$ is contained in $\operatorname{conv}\left(\iota_{V}(r V)\right)=r \operatorname{conv}\left(\iota_{V}(V)\right)$, the set $F$ corresponding to the vertices of $m+\Delta_{\sigma}$ is a maximal element of $\operatorname{esd}_{r}\left(\Delta^{d}\right)$.

Given $k$ with $0<k<r$ and $y \in \mathbb{R}^{d+1}$ we define:

$$
\alpha_{k}^{i j}(y)=\left\{\begin{array}{cc}
r\left(y_{i}-y_{j}\right)+k y_{d+1} & \text { for } 1 \leq i<j<d+1 \\
r y_{i}-k y_{d+1} & \text { for } 1 \leq i<j=d+1
\end{array}\right.
$$

The following easy and well-known result implies that the linear form $\alpha_{k}^{i j}: \mathbb{R}^{d+1} \rightarrow \mathbb{R}$ has the property that $\operatorname{conv}\left(\iota_{V}(F)\right)=m(F)+\Delta_{\sigma(F)}$ is contained in either its positive- or its negative associated halfspace for every $F \in \operatorname{esd}_{r}\left(\Delta^{d}\right)$. For a proof we refer to Knudsen [15, Lemma 2.4].

Lemma 6.2 The hyperplanes $H_{k}^{i j}=\left(\alpha_{k}^{i j}\right)^{-1}(0)$ induce a triangulation of the simplex $\operatorname{conv}\left(\iota_{V}(r V)\right)$ into simplices of the form $m+\Delta_{\sigma}$.

The above lemma in particular implies that after choosing a total order on $r V$ we have that $\left|T\left(\operatorname{esd}_{r}\left(\Delta^{d}\right)\right)\right| \cong \operatorname{conv}\left(\iota_{V}(r V)\right) \cong \Delta_{e}$ where $e \in \Sigma_{d}$ is the neutral element.

Definition 6.3 Let $\Delta$ be an abstract simplicial complex and let $r \geq 1$. Define $\operatorname{esd}_{r}(T(\Delta))$ to be the (embedded) toric complex with $\Pi_{\mathrm{esd}_{r}(T(\Delta))}=\operatorname{esd}_{r}(\Delta)$, with $\operatorname{esd}_{r}(T(\Delta))_{F}$ given by the restriction of $\iota_{V}: r V \rightarrow \mathbb{Z}^{d+1}$ to $F$ for $F \in \Pi_{\operatorname{esd}_{r}(T(\Delta))}$ and with $\operatorname{esd}_{r}(T(\Delta))_{F G}=\operatorname{id}_{\mathbb{Z}^{d+1}}$ for $F, G \in \Pi_{\mathrm{esd}_{r}(T(\Delta))}$ with $F \subseteq G$.

Define the convex function $f: \mathbb{R}^{d+1} \rightarrow \mathbb{R}$ by

$$
f(y)=\sum_{\substack{1 \leq i<j \leq d+1 \\ 0<k<r}}\left|\alpha_{k}^{i j}\left(\iota_{V}(y)\right)\right|
$$

and let $\omega=f \circ \iota_{V}: r V \rightarrow \mathbb{R}$. Lemma 6.2 implies that for every $F \in \operatorname{esd}_{r}\left(\Delta^{d}\right)$ there exists a linear form $\alpha_{F}: \mathbb{R}^{d+1} \rightarrow \mathbb{R}$ such that $\alpha_{F}(y)=f(y)$ for every $y \in m(F)+\Delta_{\sigma(F)}$. Since the convex function $f$ agrees with $\alpha_{F}$ on an open subset of $\mathbb{R}^{d+1}$ we have that $\alpha_{F}(y) \leq f(y)$ for $y \in \mathbb{R}^{d+1}$, and because $m(F)+\Delta_{\sigma(F)}$ is a simplex in the triangulation 
induced by the $H_{k}^{i j}$, equality holds precisely if $y \in \operatorname{conv}\left(\operatorname{esd}_{r}\left(T\left(\Delta^{d}\right)\right)_{F}(F)\right)$. This implies that $\operatorname{esd}_{r}\left(\Delta^{d}\right) \subseteq \Pi_{\mathrm{sd}_{\omega} r T\left(\Delta^{d}\right)}$. On the other hand if $F$ is a maximal face of $\operatorname{sd}_{\omega}\left(r T\left(\Delta^{d}\right)\right)$, then by $6.2 \operatorname{conv}\left(\operatorname{esd}_{r}\left(T\left(\Delta^{d}\right)\right)_{F}(F)\right)$ is of the form $m(F)+\Delta_{\sigma(F)}$. As in the discussion after 6.1 we see that $F \in \operatorname{esd}_{r}\left(\Delta^{d}\right)$.

Proposition 6.4 For every abstract simplicial complex $\Delta$ with vertex set $V$ we have that $\operatorname{sd}_{\omega} r T(\Delta)=\operatorname{esd}_{r}(T(\Delta))$ is a regular triangulation of $r T(\Delta)$.

Proof: We have just proved the result in the case $\Delta=\Delta^{d}$. Since $\Pi_{\mathrm{sd}_{\omega} r T(\Delta)}=\Pi_{\mathrm{sd}_{\omega} r T\left(\Delta^{d}\right)} \cap$ $\left\{F \subseteq r V: \bigcup_{u \in F} \operatorname{supp}(u) \in \Delta\right\}$ and $\Pi_{\mathrm{esd}_{r} T(\Delta)}=\Pi_{\operatorname{esd}_{r}\left(T\left(\Delta^{d}\right)\right)} \cap\left\{F \subseteq r V: \bigcup_{u \in F} \operatorname{supp}(u) \in\right.$ $\Delta\}$ it follows that $\operatorname{sd}_{\omega} r T(\Delta)=\operatorname{esd}_{r}(T(\Delta))$. Applying Corollary 5.7 we get that $\operatorname{sd}_{\omega} r T(\Delta)$ is a regular triangulation of $r T(\Delta)$.

Corollary 6.5 We have that

$$
K\left[\operatorname{esd}_{r}(\Delta)\right]=K\left[\operatorname{esd}_{r}(T(\Delta))\right]=K\left[\operatorname{sd}_{\omega}(r T(\Delta))\right] \cong K[r V] / \operatorname{in}_{\omega}\left(I_{r T(\Delta)}\right) .
$$

Proof: Only the last isomorphism does not follow directly from the definitions. By Theorem 5.11 the face ring $K\left[\operatorname{sd}_{\omega}(r T(\Delta))\right]$ is isomorphic to $K[r V] / \operatorname{rad}\left(\operatorname{in}_{\omega}\left(I_{r T(\Delta)}\right)\right)$. Since for every $F \in \Pi_{\text {sd }_{\omega} r T(\Delta)}$ the set $\iota_{V}(F)$ is a subset of the vertices of a simplex of the form $m+\Delta_{\sigma}$ for $m \in \mathbb{Z}^{d} \times\{r\}$ and $\sigma \in \Sigma_{d}$, the set $\iota_{V}(F)$ can be extended to a basis for $\mathbb{Z}^{d+1}$. By Proposition 5.13 we conclude that $\operatorname{in}_{\omega}\left(I_{r T(\Delta)}\right)$ is a radical ideal.

Let $\psi: V^{r} \rightarrow r V$ be the surjective map taking $v=\left(v_{1}, \ldots, v_{r}\right)$ to the function $\psi(v): V \rightarrow \mathbb{N}$ with $\psi(v)(i)=\left|\left\{l \in\{1, \ldots, r\}: v_{l}=i\right\}\right|$. Restricting $\psi$ to the subset $W$ of $V^{r}$ consisting of tuples of the form $v=\left(v_{1}, \ldots, v_{r}\right)$ with $1 \leq v_{1} \leq v_{2} \leq$ $\cdots \leq v_{r} \leq d+1$ we obtain a bijection $\psi: W \rightarrow r V$. Note that $\left(\iota_{V}(\psi(v))_{j}\right.$ is given by the cardinality of $\left\{l \in\{1, \ldots, r\}: 1 \leq v_{l} \leq j\right\}$ for $1 \leq j \leq d+1$. Given a subset $F$ of $W$ we have that $\psi(F) \in \operatorname{esd}_{r}(\Delta)$ if and only if the following two conditions are satisfied:

(i) there exists an ordering $F=\left\{v_{1}, \ldots, v_{s}\right\}$ of the elements of $F$ with $v_{i}=\left(v_{i 1}, \ldots, v_{i r}\right)$ $\in W$ such that

$$
1 \leq v_{11} \leq v_{21} \leq \cdots \leq v_{s 1} \leq v_{12} \leq v_{22} \leq \cdots \leq v_{1 r} \leq v_{2 r} \leq \cdots \leq v_{s r} \leq d+1,
$$

(ii) the set $\left\{v_{i j}: 1 \leq i \leq s, 1 \leq j \leq r\right\}$ is a face of $\Delta$.

Let $K[W]$ denote the polynomial ring on the set $W$. We have a natural surjection $\Phi_{r T(\Delta)}: K[W] \rightarrow K[\Delta]^{(r)}$ defined by $\Phi_{r T(\Delta)}\left(x_{\left(v_{1}, \ldots, v_{r}\right)}\right)=x_{v_{1}} \cdots x_{v_{r}}$ and we write $I_{r T(\Delta)}^{\prime}$ for the kernel of $\Phi_{r T(\Delta)}$. Note that $\Phi_{r T(\Delta)}$ induces an isomorphism $\Phi_{r T(\Delta)}: K[W] / I_{r T(\Delta)}^{\prime} \rightarrow$ $K[\Delta]^{(r)}=K[r T(\Delta)]=K[r V] / I_{r T(\Delta)}$. 
A monomial $x^{u} \in K[W]$ is of the form $x^{u}=\prod_{i=1}^{s} x_{u_{i}}$ for $u_{i}=\left(u_{i 1}, \ldots, u_{i r}\right) \in W$. There exists a unique matrix of the form

$$
\left(\begin{array}{cccc}
v_{11} & v_{12} & \cdots & v_{1 r} \\
v_{21} & v_{22} & \cdots & v_{2 r} \\
\vdots & \vdots & \vdots & \vdots \\
v_{s 1} & v_{s 2} & \cdots & v_{s r}
\end{array}\right)
$$

with

$$
1 \leq v_{11} \leq v_{21} \leq \cdots \leq v_{s 1} \leq v_{12} \leq v_{22} \leq \cdots \leq v_{1 r} \leq v_{2 r} \leq \cdots \leq v_{s r} \leq d+1 .
$$

and $\prod_{i=1}^{s} \prod_{j=1}^{r} x_{u_{i j}}=\prod_{i=1}^{s} \prod_{j=1}^{r} x_{v_{i j}} \in K[V]$. Let $\operatorname{sort}\left(x^{u}\right)=\prod_{i=1}^{s} x_{\left(v_{i 1}, \ldots, v_{i r}\right)}$. Motivated by Sturmfels [23, 14.2] we call the monomial $x^{u} \in K[W]$ sorted if $x^{u}=\operatorname{sort}\left(x^{u}\right)$.

If $x^{u}=x_{\left(u_{11}, \ldots, u_{1 r}\right)} x_{\left(u_{21}, \ldots, u_{2 r}\right)}$, then $\psi\left(u_{11}, \ldots, u_{1 r}\right)$ and $\psi\left(u_{21}, \ldots, u_{2 r}\right)$ are connected by an edge in $\operatorname{esd}_{r}\left(T\left(\Delta^{d}\right)\right)$ if and only if $x^{u}$ is sorted.

Let $\omega^{\prime}=\omega \circ \psi: W \rightarrow \mathbb{R}$ where $\omega=f \circ \iota_{V}$ as above. The following is essentially Theorem 14.2 in Sturmfels [23] (see Hibi-Ohsugi [17] for related Gröbner bases).

Proposition 6.6 The initial ideal $\operatorname{in}_{\omega^{\prime}}\left(I_{r T\left(\Delta^{d}\right)}^{\prime}\right)$ is generated by the initial polynomials of the set $\mathcal{G}$ consisting of the binomials

$$
x_{\left(u_{11}, \ldots, u_{1 r}\right)} x_{\left(u_{21}, \ldots, u_{2 r}\right)}-\operatorname{sort}\left(x_{\left(u_{11}, \ldots, u_{1 r}\right)} x_{\left(u_{21}, \ldots, u_{2 r}\right)}\right) .
$$

The initial polynomial of $x^{u}-\operatorname{sort}\left(x^{u}\right)$ is $x^{u}$.

Proof: Since the case $r=1$ is trivial, assume $r \geq 2$. Observe that by the discussion above for every non-face $F \subseteq r V$ of $\operatorname{esd}_{r}\left(\Delta^{d}\right)$ there exists a non-face $G \subseteq F$ of $\operatorname{esd}_{r}\left(\Delta^{d}\right)$ with $|G|=2$.

Since $K[\psi]: K[W] \rightarrow K[r V]$ takes $I_{r T\left(\Delta^{d}\right)}^{\prime}$ to $I_{r T\left(\Delta^{d}\right)}$, it follows from Corollary 6.5 that $\operatorname{in}_{\omega^{\prime}}\left(I_{r T\left(\Delta^{d}\right)}^{\prime}\right)$ is generated by square-free quadratic monomials of the form $x_{\left(u_{11}, \ldots, u_{1 r}\right)}$ $x_{\left(u_{21}, \ldots, u_{2 r}\right)}$ where $\left\{\psi\left(u_{11}, \ldots, u_{1 r}\right), \psi\left(u_{21}, \ldots, u_{2 r}\right)\right\}$ is not a face in $\operatorname{esd}_{r}\left(\Delta^{d}\right)$, that is, the vertices $\psi\left(u_{11}, \ldots, u_{1 r}\right)$ and $\psi\left(u_{21}, \ldots, u_{2 r}\right)$ are not connected by an edge in $\operatorname{esd}_{r}\left(T\left(\Delta^{d}\right)\right)$. It follows that $x_{\left(u_{11}, \ldots, u_{1 r}\right)} x_{\left(u_{21}, \ldots, u_{2 r}\right)} \in \operatorname{in}_{\omega^{\prime}}\left(I_{r T\left(\Delta^{d}\right)}^{\prime}\right)$ if and only if there exists $i, j, k$ such that the real numbers $\alpha_{k}^{i j}\left(\iota_{V}\left(\psi\left(u_{11}, \ldots, u_{1 r}\right)\right)\right)$ and $\alpha_{k}^{i j}\left(\iota_{V}\left(\psi\left(u_{21}, \ldots, u_{2 r}\right)\right)\right)$ are non-zero and have opposite signs. Therefore

$$
\begin{aligned}
& \omega^{\prime}\left(u_{11}, \ldots, u_{1 r}\right)+\omega^{\prime}\left(u_{21}, \ldots, u_{2 r}\right) \\
& \quad=\omega\left(\psi\left(u_{11}, \ldots, u_{1 r}\right)\right)+\omega\left(\psi\left(u_{21}, \ldots, u_{2 r}\right)\right) \\
& \quad>\omega\left(\psi\left(u_{11}, \ldots, u_{1 r}\right)+\psi\left(u_{21}, \ldots, u_{2 r}\right)\right)
\end{aligned}
$$


On the other hand, since $x_{\left(v_{11}, \ldots, v_{1 r}\right)} x_{\left(v_{21}, \ldots, v_{2 r}\right)}=\operatorname{sort}\left(x_{\left(u_{11}, \ldots, u_{1 r}\right)} x_{\left(u_{21}, \ldots, u_{2 r}\right)}\right)$ is sorted we have that

$$
\omega^{\prime}\left(v_{11}, \ldots, v_{1 r}\right)+\omega^{\prime}\left(v_{21}, \ldots, v_{2 r}\right)=\omega\left(\psi\left(v_{11}, \ldots, v_{1 r}\right)+\psi\left(v_{21}, \ldots, v_{2 r}\right)\right) .
$$

It follows that $x_{\left(u_{11}, \ldots, u_{1 r}\right)} x_{\left(u_{21}, \ldots, u_{2 r}\right)}-x_{\left(v_{11}, \ldots, v_{1 r}\right)} x_{\left(v_{21}, \ldots, v_{2 r}\right)} \in I_{r T\left(\Delta^{d}\right)}^{\prime}$ with initial polynomial $x_{\left(u_{11}, \ldots, u_{1 r}\right)} x_{\left(u_{21}, \ldots, u_{2 r}\right)}$. This concludes the proof.

Next we deal with an arbitrary abstract simplicial complex $\Delta$.

Theorem 6.7 The ideal $\operatorname{in}_{\omega^{\prime}}\left(I_{r T(\Delta)}^{\prime}\right)$ is generated by the initial polynomials of the union $\mathcal{G}^{\prime}$ of the binomials in $\mathcal{G}$ and the sorted square-free monomials $x^{u}$ with $\psi(\operatorname{supp}(u))$ a non-face of $\operatorname{esd}_{r}(\Delta)$.

Proof: The bijection $\psi: W \rightarrow r V$ induces an isomorphism $\psi: K[W] \rightarrow K[r V]$ with $\psi\left(x^{u}\right)=\prod_{w \in W} x_{\psi(w)}^{u(w)}$. It follows from 4.2 that

$$
\begin{aligned}
& K[W] / \operatorname{in}_{\omega^{\prime}}\left(I_{r T(\Delta)}^{\prime}\right) \\
& \quad \cong K[r V] / \operatorname{in}_{\omega}\left(I_{r T(\Delta)}\right) \\
& \quad=K[r V] / \operatorname{in}_{\omega}\left(I_{r T\left(\Delta^{d}\right)}\right)+\left(\psi\left(x^{u}\right): \operatorname{supp}\left(\psi\left(x^{u}\right)\right) \notin \Pi_{\operatorname{sd}_{\omega} r T(\Delta)}\right) \\
& \quad \cong K\left[\operatorname{esd}_{r}\left(\Delta^{d}\right)\right] /\left(\psi\left(x^{u}\right): x^{u} \text { square-free and sorted, } \operatorname{supp}\left(\psi\left(x^{u}\right)\right) \notin \Pi_{\operatorname{sd}_{\omega} r T(\Delta)}\right) .
\end{aligned}
$$

Observe that for a sorted monomial $x^{u}=\prod_{i=1}^{s} x_{\left(u_{i 1}, \ldots, u_{i r}\right)} \in K[W]$ we have that $\operatorname{supp}\left(\psi\left(x^{u}\right)\right)$ $\notin \Pi_{\mathrm{sd}_{\omega} r T(\Delta)}$ if and only if $\left\{u_{i r}: 1 \leq i \leq s, 1 \leq j \leq r\right\} \notin \Delta$. This is exactly the case if $\operatorname{supp}\left(\psi\left(x^{u}\right)\right)$ is not a face of $\operatorname{esd}_{r}(\Delta)=\Pi_{\mathrm{sd}_{\omega}} r T(\Delta)$. Hence

$$
K[W] / \operatorname{in}_{\omega^{\prime}}\left(I_{r T(\Delta)}^{\prime}\right) \cong K\left[\operatorname{esd}_{r}(\Delta)\right]
$$

For the rest of this section $K$ denotes a field. For a $\mathbb{Z}$-graded $K$-algebra $R$ we denote with $H(R, n)=\operatorname{dim}_{K} R_{n}$ for $n \in \mathbb{Z}$ the Hilbert function of $R$. If $R=K[W] / L$ for a graded ideal $L \subset K[W]$ and for a finitely generated graded module $M$ we denote with proj $\operatorname{dim}_{R}(M)=\sup \left\{i \in \mathbb{N}\right.$ : $\left.\operatorname{Tor}_{i}^{K[W]}(R, K) \neq 0\right\}$ the projective dimension of $M$ and with $\operatorname{reg}_{R}(M)=\sup \left\{j \in \mathbb{Z}: \operatorname{Tor}_{i}^{K[W]}(R, K)_{i+j} \neq 0\right.$ for some $\left.i \geq 0\right\}$ the Castelnuovo-Mumford regularity of $R$. A standard graded $K$-algebra $R$ is said to be Koszul if $\operatorname{reg}_{R}(K)=0$ where $K$ is regarded as an $R$-module. For the Cohen-Macaulay and Gorenstein property of rings see for example [6]. The next corollary lists some algebraic consequences for the face rings.

Corollary 6.8 Let $\Delta$ be an abstract simplicial complex on $\{1, \ldots, d+1\}$ and let $K$ be a field. We have:

(i) $\operatorname{Hilb}\left(K\left[\operatorname{esd}_{r}(\Delta)\right], n\right)=\operatorname{Hilb}\left(K[\Delta]^{(r)}, n\right)$ for $n \geq 0$.

(ii) $K[\Delta]^{(r)}$ is Cohen-Macaulay, Gorenstein or Koszul if $K\left[\operatorname{esd}_{r}(\Delta)\right]$ has one of these properties. 
(iii) $\operatorname{proj}_{\operatorname{dim}_{K[W]}}\left(K\left[\operatorname{esd}_{r}(\Delta)\right]\right) \geq \operatorname{proj} \operatorname{dim}_{K[W]}\left(K[\Delta]^{(r)}\right)$.

(iv) $\operatorname{reg}_{K[W]}\left(K\left[\operatorname{esd}_{r}(\Delta)\right]\right) \geq \operatorname{reg}_{K[W]}\left(K[\Delta]^{(r)}\right)$.

Proof: Using the fact that every weight order $\omega$ can be refined to a monomial order this follows from standard arguments. See for example Bruns and Conca [4].

Remark 6.9 By a theorem of Backelin and Fröberg [1] (see also Eisenbud, Reeves and Totaro [9]) one knows that the $r$-th Veronese algebra of $K[\Delta]$ is Koszul for $r \gg 1$. One could hope that this property is inherited for $\operatorname{esd}_{r}(\Delta)$ for $r \gg 1$. This is however not the case. Let for example $\Delta$ be the set of subsets $F \subset\{1,2,3,4\}$ such that $F \neq\{1,2,3,4\}$. Then $x_{(1,4,4, \ldots)} \cdot x_{(2,4,4, \ldots)} \cdot x_{(3,4,4, \ldots)}=0$ in $K\left[\operatorname{esd}_{r}(\Delta)\right]$. But the elements $x_{(1,4,4, \ldots)} \cdot x_{(2,4,4, \ldots)}$, $x_{(1,4,4, \ldots)} \cdot x_{(3,4,4, \ldots)}$ and $x_{(2,4,4, \ldots)} \cdot x_{(3,4,4, \ldots)}$ are all non-zero in $K\left[\operatorname{esd}_{r}(\Delta)\right]$. Hence $x_{(1,4,4, \ldots)}$. $x_{(2,4,4, \ldots)} \cdot x_{(3,4,4, \ldots)}$ belongs to a minimal system of generators for the defining ideal of $\operatorname{esd}_{r}(\Delta)$, but is not a quadratic monomial. The Koszul property would imply that the defining ideal of $\operatorname{esd}_{r}(\Delta)$ is generated by quadratic monomials. Hence $K\left[\operatorname{esd}_{r}(\Delta)\right]$ is not Koszul for any $r \geq 1$.

\section{References}

1. J. Backelin and R. Fröberg, "Koszul algebras, Veronese subrings and rings with linear resolutions," Rev. Roum. Math. Pures Appl. 30 (1985), 85-97.

2. M. Bökstedt, W.C. Hsiang, and I. Madsen, "The cyclotomic trace and algebraic K-theory of spaces," Invent. Math. 111 (1993), 465-539.

3. L.A. Borisov, L. Chen, and G.G. Smith, "The orbifold Chow ring of toric Deligne-Mumford stacks," J. Am. Math. Soc. 18 (2005), 193-215.

4. W. Bruns and A. Conca, "Groebner bases, initial ideals and initial algebras." In L.L. Avramov et al. (Hrsg.), Homological methods in commutative algebra, IPM Proceedings, Teheran 2004

5. W. Bruns and J. Gubeladze, "Polyhedral algebras, arrangements of toric varieties, and their groups. Computational commutative algebra and combinatorics," Adv. Stud. Pure Math. 33 (2001), 1-51.

6. W. Bruns and J. Herzog, Cohen-Macaulay Rings. Rev. ed., Cambridge Studies in Advanced Mathematics 39 , Cambridge University Press (1998)

7. D.A. Cox, "The homogeneous coordinate ring of a toric variety," J. Algebr. Geom. 4 (1995), 17-50.

8. H. Edelsbrunner and D.R. Grayson, "Edgewise subdivision of a simplex," Discrete Comput. Geom. 24 (2000), 707-719.

9. D. Eisenbud, A. Reeves, and B. Totaro, "Initial ideals, Veronese subrings, and rates of algebras," Adv. Math. 109 (1994), 168-187.

10. D. Eisenbud and B. Sturmfels, "Binomial ideals," Duke Math. J. 84 (1996), 1-45.

11. H. Freudenthal, "Simplizialzerlegungen von beschränkter Flachheit," Ann. Math. 43 (1942), 580-582.

12. W. Fulton, "Introduction to Toric Varieties," Annals of Mathematics Studies, Princeton University Press, 1993, vol. 131 .

13. D. Grayson, "Exterior power operations on higher K-theory," K-Theory 3 (1989), 247-260.

14. S. Hosten, D. MacLagan, and B. Sturmfels, "Supernormal vector configurations," J. Algebr. Comb. 19 (2004), 297-313.

15. G. Kempf, F. Knudsen, D. Mumford, and B. Saint-Donat, Toroidal Embeddings. I. Lecture Notes in Mathematics, Springer, 1973, vol. 339.

16. D. Notbohm and N. Ray, "On Davis-Januszkiewicz homotopy types I; formality and rationalisation," Algebr. Geom. Topol. 5 (2005), 31-51.

17. H. Ohsugi and T. Hibi, "Compressed polytopes, initial ideals and complete multipartite graphs," Ill. J. Math. 44 (2000), 391-406. 
18. A. Schrijver, Theory of Linear and Integer Programming, Wiley, 1998.

19. E. O'Shea and R.R. Thomas, "Toric initial ideals of $\Delta$-normal configurations: Cohen-Macaulayness and degree bounds," J. Algebr. Comb. 21 (2005), 247-268.

20. R.P. Stanley, Combinatorics and Commutative Algebra. 2nd ed., Progress in Mathematics Birkhäuser, 1996, vol. 41.

21. R. Stanley, "Generalized $h$-vectors, intersection cohomology of toric varieties, and related results. Commutative algebra and combinatorics," Adv. Stud. Pure Math. 11 (1987), 187-213.

22. B. Sturmfels, "Gröbner bases of toric varieties," Tôhoku Math. J. 43 (1991), 249-261.

23. B. Sturmfels, "Gröbner bases and convex polytopes," Univ. Lecture Series 8, AMS, 1996.

24. G.M. Ziegler, Lectures on polytopes. Graduate Texts in Mathematics, Springer, 1995, vol. 152. 